\title{
Akirin Links Twist-Regulated Transcription with the Brahma Chromatin Remodeling Complex during Embryogenesis
}

\author{
Scott J. Nowak ${ }^{1 \times a}$, Hitoshi Aihara ${ }^{2 \times b}$, Katie Gonzalez ${ }^{3 \times c}$, Yutaka Nibu ${ }^{2,3}$, Mary K. Baylies ${ }^{1,3 *}$ \\ 1 Program in Developmental Biology, Sloan Kettering Institute, New York, New York, United States of America, 2 Cell and Developmental Biology, Weill Cornell Medical \\ College, New York, New York, United States of America, 3 Weill Cornell Graduate School of Biomedical Sciences, Cornell University, New York, New York, United States of \\ America
}

\begin{abstract}
The activities of developmentally critical transcription factors are regulated via interactions with cofactors. Such interactions influence transcription factor activity either directly through protein-protein interactions or indirectly by altering the local chromatin environment. Using a yeast double-interaction screen, we identified a highly conserved nuclear protein, Akirin, as a novel cofactor of the key Drosophila melanogaster mesoderm and muscle transcription factor Twist. We find that Akirin interacts genetically and physically with Twist to facilitate expression of some, but not all, Twist-regulated genes during embryonic myogenesis. akirin mutant embryos have muscle defects consistent with altered regulation of a subset of Twistregulated genes. To regulate transcription, Akirin colocalizes and genetically interacts with subunits of the Brahma SWI/SNFclass chromatin remodeling complex. Our results suggest that, mechanistically, Akirin mediates a novel connection between Twist and a chromatin remodeling complex to facilitate changes in the chromatin environment, leading to the optimal expression of some Twist-regulated genes during Drosophila myogenesis. We propose that this Akirin-mediated link between transcription factors and the Brahma complex represents a novel paradigm for providing tissue and target specificity for transcription factor interactions with the chromatin remodeling machinery
\end{abstract}

Citation: Nowak SJ, Aihara H, Gonzalez K, Nibu Y, Baylies MK (2012) Akirin Links Twist-Regulated Transcription with the Brahma Chromatin Remodeling Complex during Embryogenesis. PLoS Genet 8(3): e1002547. doi:10.1371/journal.pgen.1002547

Editor: Eric Rulifson, University of California San Francisco, United States of America

Received February 2, 2011; Accepted January 4, 2012; Published March 1, 2012

Copyright: (c) 2012 Nowak et al. This is an open-access article distributed under the terms of the Creative Commons Attribution License, which permits unrestricted use, distribution, and reproduction in any medium, provided the original author and source are credited.

Funding: This work was supported by the Sloan-Kettering Institute and NIH grants GM056989 and GM078318 to MKB and by a Research Scholar Grant (RSG-08042-01-DDC) from the American Cancer Society to YN. The funders had no role in study design, data collection and analysis, decision to publish, or preparation of the manuscript.

Competing Interests: The authors have declared that no competing interests exist.

*E-mail: m-baylies@ski.mskcc.org

wa Current address: Department of Biology and Physics, Kennesaw State University, Kennesaw, Georgia, United States of America

ab Current address: Department of Biochemistry, Nagasaki University Graduate School of Biomedical Sciences, Nagasaki, Japan

ac Current address: Molecular Diagnostic Services, San Diego, California, United States of America

\section{Introduction}

A fundamental question in embryonic development is how diverse cell lineages are specified, patterned and organized from a single common progenitor. These processes are governed by distinct gene expression programs administered by tightly regulated transcription factor activities during embryonic development. One mechanism whereby transcription factor activities are modulated is through direct interactions with cofactors. In addition, links between transcription factors and the general transcription machinery can be indirect, such as the actions of secondary factors that recruit chromatin remodeling complexes to modify the local chromatin environment and allow gene activation. The identification of these secondary effectors and their in vivo function is critical for understanding the regulation of transcription factor activity during embryonic development. Such interactions have significant ramifications for developmental disorders and diseases such as cancer.

The Twist transcription factor represents an ideal model for studying the regulation of transcription factor activity throughout development. Twist is a highly conserved transcription factor that is a key regulator of many developmental programs during embryogenesis as well as cancer metastasis [1-3]. In Drosophila melanogaster, Twist regulates multiple, discrete steps of mesoderm development, including gastrulation, segregation of populations of mesodermal cells, establishment and formation of the somatic musculature, and finally establishment of the adult musculature [4-7]. To achieve these diverse activities, Twist regulates a large number of target genes temporally within a discrete lineage resulting in a diverse array of outputs during mesodermal development [8]. A key question is how these diverse outputs of Twist activity during development are achieved.

The varied roles of Twist during different phases of embryonic development and the large number of Twist-regulated target genes suggest complex regulation of Twist activity $[4,8,9]$. Twist activity is often modulated by interactions with other transcription factors [10,11]. Twist is a basic Helix-loop-Helix (bHLH) transcription factor that can homodimerize or heterodimerize, with distinct activities for each dimer pair. For example, Twist homodimers are responsible for activating target genes that direct cells to the somatic myogenic lineage [10]. By contrast, heterodimers between Twist and another bHLH protein, Daughterless, repress the somatic 


\section{Author Summary}

The proper development of the diverse array of cell types in an organism depends upon the induction and repression of specific genes at particular times and places. This gene regulation requires both the activity of tissuespecific transcriptional regulators and the modulation of the chromatin environment. To date, a complete picture of the interplay between these two processes remains unclear. To address this, we examined the activity of the evolutionarily conserved transcription factor Twist during embryogenesis of Drosophila melanogaster. While Twist has multiple activities and roles during development, a direct link between Twist and chromatin remodeling is unknown. We identified a highly conserved protein, Akirin, as a link between Twist and chromatin remodeling factors. Akirin is required for optimal expression of a Twistdependent target during muscle development via interactions with the Drosophila SWI/SNF chromatin remodeling complex. Interestingly, Akirin is not required for activation of all Twist-dependent enhancers, suggesting that Akirin refines Twist activity outputs and that different Twist-dependent targets have different requirements for chromatin remodeling during development. Our data further suggests that Akirin similarly links the SWI/SNF chromatin remodeling complex with other transcription factors during development. This work has important ramifications for understanding both normal development and diseases such as cancer.

myogenic lineage [10,11]. Twist activity is also regulated by interactions with other transcription regulators that bind closely linked DNA regulatory elements. One such interaction, with the $\mathrm{NF}-\kappa \mathrm{B}$ orthologue Dorsal, produces synergistic rather than cooperative activation of Dorsal targets during development [1214]. Hence, Daughterless and Dorsal are examples of transcription regulators that physically interact with Twist to modify its output.

Other mechanisms that regulate Twist activity during development are less clear. One mechanism whereby transcriptional activity is indirectly regulated is through the action of chromatin modifying factors that generate a local chromatin environment that allows and/or favors the expression of a specific target gene. The structure of chromatin is modified by two predominant mechanisms: the marking of nucleosome tails with post-translational modifications such as acetylation, phosphorylation and methylation, and the remodeling of the local chromatin environment via factors that reposition nucleosomes in a local gene environment [15]. Nucleosome repositioning occurs via the activity of ATP-dependent chromatin remodeling complexes such as the Brahma-containing (BRM) complex, the Drosophila orthologue of the yeast SWI/SNF chromatin remodeling complex $[15,16]$. Mutations in BRM complex subunits have revealed essential roles for BRM in such processes as homeotic gene expression, oogenesis, and cell cycle control [17-22]. Loss-offunction studies have determined that BRM-regulated chromatin remodeling activity is required for most RNA Polymerase IIregulated transcription [23]. Most eukaryotic organisms have two different compositions of the SWI/SNF complex; in Drosophila, these complexes are designated the BAP (Osa-containing) and PBAP (polybromo/Bap180, Bap170 and SAYP-containing) complexes $[15,16,24]$. Both $\mathrm{BAP}$ and PBAP are linked to gene activation and repression, are present in the same cells, and perform unique yet cooperative functions during development [19,24-28]. Both the association of BRM complexes with transcription factors and BRM complex targeting and regulation during embryogenesis remain an area of considerable interest. Moreover, links between BRM complexes, chromatin remodeling and Twist have not yet been elucidated.

The nuclear protein Akirin was shown to regulate gene expression in several different transcription pathways, yet its mechanism of action remained unclear [29,30]. Here we identify Akirin as a factor that facilitates an interaction between Twist and the BRM chromatin remodeling complex to promote gene expression. We find that Akirin interacts both physically and genetically with Twist at Twist-dependent enhancer regions and positively regulates expression of Dmef2, a Twist-regulated gene that is critical for somatic myogenesis during development. As would be predicted by this interaction, akirin mutant embryos show a range of somatic muscle phenotypes. Akirin is widely associated with regions of active transcription. We find that Akirin colocalizes with subunits of the BRM chromatin remodeling complex on polytene chromosomes and interacts genetically with core subunits of the BRM complex during myogenesis. Finally, we verify with chromatin immunoprecipitation that Twist, Akirin, and a core subunit of the BRM complex all occupy the Twist-dependent Dmef2 enhancer. Curiously, Akirin/Twist interactions are not required at all Twist-dependent enhancers, as evidenced by our chromatin immunoprecipitation experiments at the eve MHE. These results suggest that Akirin functions as a BRM accessory protein that links the BRM chromatin remodeling machinery to Twist transcription factor activity at a subset of Twist-regulated enhancers. These results provide a common mechanism by which Akirin links chromatin remodeling factors to spatiotemporalspecific gene activation.

\section{Results}

Akirin is a conserved Twist-interacting nuclear protein

To identify proteins that interact with Twist, we performed a yeast double interaction screen [31,32]. Two Twist-regulated enhancers from the Dmef2 [33] and tinman [34] genes were cloned upstream of a HIS3 reporter. Yeast strains containing these enhancer/reporter constructs were first transformed with a Twist expression vector and then with a 0-6 hr embryonic cDNA library fused to a Gal4 activation domain. Transformants were positively scored for cDNAs that activated HIS3 expression at Dmef2, tinman, or both enhancers in a Twist-dependent manner. We identified 28 cDNAs that activated expression from both enhancers. From this group, we selected the then-unknown gene CG8580 for further study. CG8580 was found to require the presence of Twist for activation of the HIS3 reporter [32].

We initially named CG8580 as bhringi [32], but recent groups have re-designated this gene and its orthologues as akirin [29,35]. For clarity, we have adopted this nomenclature. The Drosophila akirin gene encodes a highly conserved 201-residue protein with orthologues present in over 24 different metazoan genomes [35]. Aside from a predicted nuclear localization sequence, Drosophila Akirin has no known protein motifs [35]. We raised antibodies against a peptide sequence found in the $\mathrm{N}$-terminal region of Drosophila Akirin (Figure S1). Immunofluorescence staining of embryos at various stages of development showed that Akirin is ubiquitously expressed and found in the nuclei of all cells of the developing embryo (Figure S2).

\section{akirin mutants display a range of muscle phenotypes}

The somatic muscles of Drosophila embryos are arranged in a stereotypic, repeated pattern of 30 muscles within each abdominal hemisegment (Figure 1) [36]. Because Akirin interacted with Twist, a transcription factor that is essential to the proper 

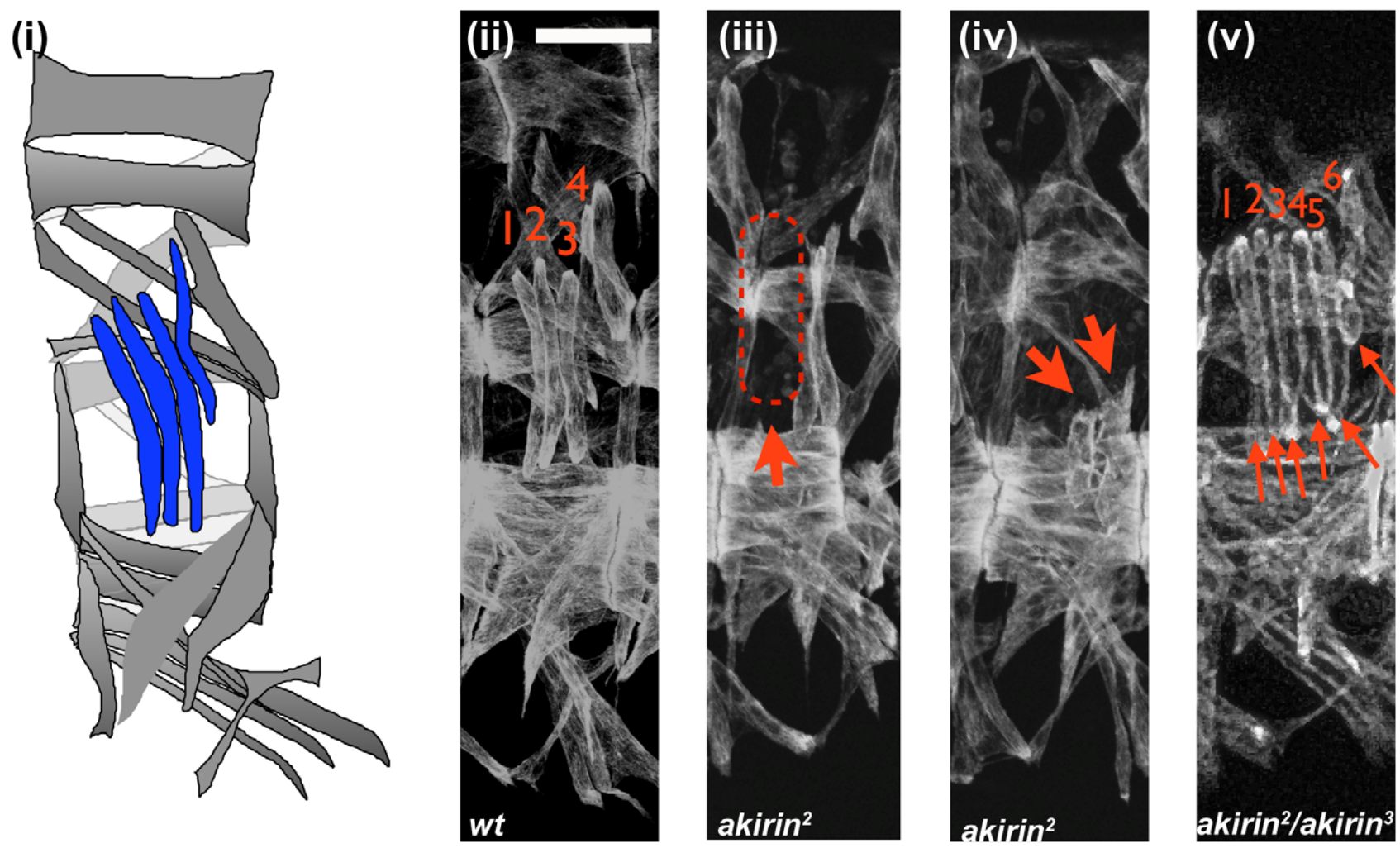

Figure 1. Akirin mutants display a range of muscle phenotypes. Lateral views of stage 16 wild-type (ii, wt) and akirin mutant (iii, iv, v) embryos stained with anti-tropomyosin antibodies demonstrate the types of muscle phenotypes observed. All allelic combinations are listed as maternal/paternal contribution. In all figures, anterior is to left and dorsal is up, scale bar $=25 \mu \mathrm{m}$. Lateral transverse (LT) muscles are used to illustrate the following predominant muscle defects observed in akirin mutants (red arrows): (iii) missing muscles, (iv) improperly attached muscles, and (v) duplicated muscles. Schematic of wild-type muscle pattern is shown in (i). Penetrance of each defect is given in Table 1. doi:10.1371/journal.pgen.1002547.g001

patterning of the somatic musculature, we examined the somatic musculature of akirin mutant embryos, using publicly available and newly generated akirin alleles (See Materials and Methods and Figure S3). akirin mutant embryos exhibit a range of somatic body wall muscle phenotypes. Three classes of defects were observed: missing muscles, attachment defects, and duplicated muscles (Figure 1). While these defects were seen easily in the 4 lateral transverse (LT) muscles, the same classes of defects were detected in several other muscles, particularly the DT1, DO3, DO4 and DA3 muscles. Misattached, missing or duplicated muscles were found in stage 16 (15 h after egg laying (AEL)) akirin ${ }^{2}$ homozygous mutant embryos $(46.5 \%, \mathrm{n}=114$ embryos), and embryos from crosses of akirin ${ }^{2}$ females with akirin ${ }^{3}$ males ( $67 \%, \mathrm{n}=52$ embryos), or akirin ${ }^{3}$ females with akirin ${ }^{5}(44.7 \%, \mathrm{n}=38$ embryos) males (Table 1). The low penetrance of muscle phenotypes seen in akirin mutants is likely due to the high degree of maternal loading of akirin RNA (Figure S3). We attempted to generate akirin maternal/ zygotic mutants by making germline clones using akirin ${ }^{3}$ mutants [37]. However, no akirin ${ }^{M Z}$ mutant embryos were recovered, suggesting that Akirin is important for oocyte formation (data not shown). Therefore, homozygous viable akirin ${ }^{2}$ females likely produce embryos that are more highly sensitized to low levels of akirin mRNA in the oocyte. Importantly, the missing muscle phenotype is not due to a failure to properly specify muscle founder cells, as immunohistochemistry of founder cell identity proteins, including Slouch, Even-skipped and Krüppel, did not show a decrease in the numbers of founder cells in akirin mutants (Figure S4). Also, in akirin mutant embryos there were no obvious signs of muscle degeneration or late differentiation defects [38] (data not shown). Epidermal development was normal, and the cuticle developed normally (data not shown).

Akirin interacts both genetically and physically with Twist

The muscle phenotypes in akirin mutant embryos were reminiscent of those observed in embryos in which twist expression is modified by RNAi in the during muscle fiber formation [11]. To determine if akirin interacts genetically in vivo with twist, we examined the patterning of the somatic body wall musculature in embryos that are heterozygous for both twist and akirin. While twi $^{1} /+$ embryos [39], akirin $/+$ and akirin $^{3} /+$ embryos have a wildtype somatic muscle pattern (data not shown), stage 16 embryos that are heterozygous for both twist and akirin ${ }^{2}$ and akirin ${ }^{3}\left(\right.$ twi $^{1} /+$; akirin $^{2} /+$, and twil $/+$; akirin $^{3} /+$ ) show a general disruption of the muscle pattern, with missing and misattached muscles $\left(t w i^{I 1} /+\right.$; akirin $^{2} /+$ : $21.6 \%$ of 60 embryos examined and twi $^{1} /+$; akirin $^{3} /+$ : $18.5 \%$ of 105 embryos examined, Figure 2A).

Additionally, in vitro-translated Akirin was successfully pulled down using a GST-Twist fusion protein indicating a physical interaction between Akirin and Twist (Figure 2B). Taken together, these data confirmed our initial results of the yeast double interaction screen and demonstrated both a physical and functional interaction between Akirin and Twist.

\section{Dmef2 enhancer activity is reduced in akirin mutant embryos}

Akirin was initially identified in our screen as a Twist interacting protein in the context of the Dmef2 enhancer. In addition, the 
Table 1. Penetrance of muscle phenotypes in akirin mutant embryos.

\begin{tabular}{|c|c|c|c|c|c|}
\hline Genotype & Embryos examined & Penetrance $^{a}$ & Missing muscles ${ }^{b}$ & Attachment defects ${ }^{b}$ & Duplicated muscles $^{b}$ \\
\hline akirin $^{2}$ & 114 & $46.5 \%$ & $67.9 \%$ & $83.0 \%$ & $18.9 \%$ \\
\hline akirin $^{2} /$ akirin $^{3}$ & 52 & $67.0 \%$ & $71.4 \%$ & $68.5 \%$ & $25.7 \%$ \\
\hline akirin $^{3} /$ akirin $^{5}$ & 38 & $44.7 \%$ & $35.0 \%$ & $35.0 \%$ & $6.0 \%$ \\
\hline wild-type & 85 & $2.3 \%$ & $1.1 \%$ & $1.1 \%$ & $0.0 \%$ \\
\hline
\end{tabular}

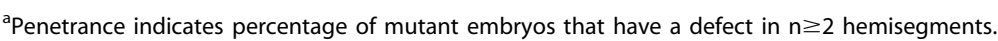

brevalence of missing, attachment, and duplication defects are given as percentage of observed defect in mutant embryos.

doi:10.1371/journal.pgen.1002547.t001

muscle defects in akirin mutants are similar to the phenotypes in embryos with modified Dmef2 or twist expression [7,11,40-42]. These data suggested that Akirin and Twist interact to positively regulate transcription from Dmef2. To test this in vivo, we analyzed akirin mutant embryos that carry a lacZ transgene regulated by the same Dmef2 muscle enhancer [33] used in our initial double interaction screen. This particular enhancer requires Twist activity for early Dmef2 expression during somatic myogenesis. Regulation of Dmef2 by Twist is critical for the subsequent establishment of the somatic musculature $[8,33,40,41,43-48]$. Whole-mount antibody staining for $\beta$-galactosidase $(\beta$-gal) indicated that akirin mutants have reduced expression of $\beta$-gal compared to wild-type embryos with the same transgene at the same developmental stage (Figure 3A, 3B). Densitometric analysis of Western blotting supported the observation that total $\beta$-gal levels were changed in akirin mutant extracts relative to wild-type embryo extracts (Figure S5). To confirm that endogenous Dmef2 expression levels were affected, we performed RT-qPGR analysis on total mRNA prepared from wild-type and akirin ${ }^{2}$ mutant embryos at the same developmental age (4-6 h AEL). Accordingly, we observed a 2.75fold reduction in Dmef2 transcripts in akirin ${ }^{2}$ mutant embryos compared to wild-type embryos at this time (Figure 3C).

For comparison, we examined a second Twist target gene, evenskipped. No reduction in protein levels of Even-skipped (Eve) or levels of expression from eveMHE-lacz reporter constructs were observed in akirin mutant embryos at the same stage (Figure 3B and Figure S5). These data indicated that Twist and Akirin interact to positively regulate expression of the Dmef2 reporter. These data also suggested that not all Twist target genes and/or their associated enhancer elements require Akirin activity for their proper expression.

\section{Akirin protein is detected at actively transcribed gene loci}

In addition to our observed relationship with Twist, Akirin has been linked to other transcriptional regulators in various insect and mouse contexts $[29,30,49]$. However, in each of these contexts, the mechanism by which Akirin promotes gene expression remained unclear. Because Akirin does not have a consensus DNA-binding domain or predicted catalytic activity, we hypothesized that Akirin regulates gene expression through its interactions with transcriptional regulators and would therefore localize to regions of active transcription. We analyzed the distribution of Akirin on polytene chromosomes and found Akirin localization throughout the genome including puffed regions of polytene chromosomes (Figure 4A, 4B), which indicate active transcription [50]. For further confirmation, co-immunostaining for Akirin and Serine 10-phosophorylated histone H3, a histone
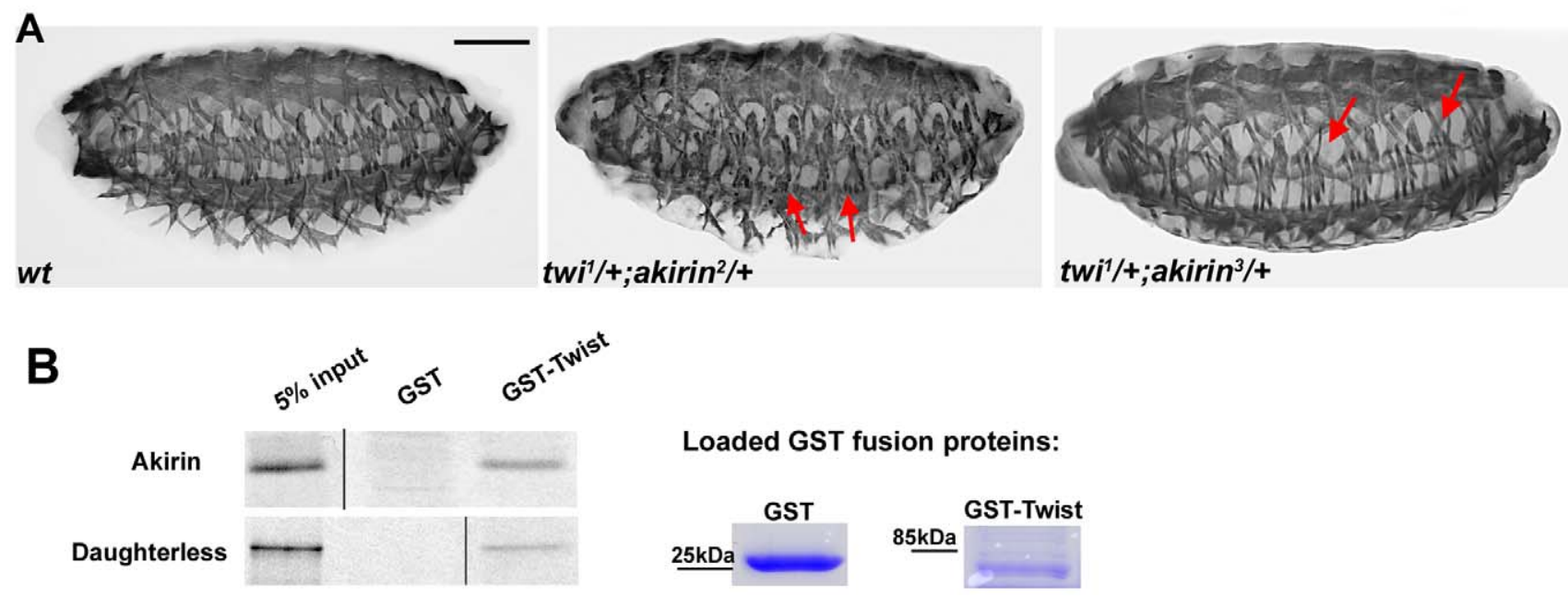

Loaded GST fusion proteins:

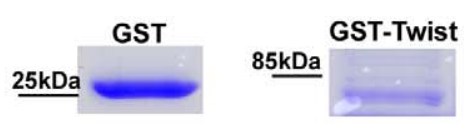

Figure 2. Akirin interacts both genetically and physically with Twist. (A) Lateral views of stage 16 wild-type (wt) and embryos heterozygous for both twist and akirin (twi ${ }^{1} /+$; akirin ${ }^{2} /+$ and $t^{2} i^{1} /+$; akirin ${ }^{3} /+$ ) stained with tropomyosin antibodies to visualize body wall muscles. Double heterozygous embryos have defects in muscle patterning (indicated by red arrows), suggesting a genetic interaction between akirin and twist. Scale $\mathrm{bar}=50 \mu \mathrm{m}$. (B) GST pull-down experiments indicate that GST-Twist interacts physically with in vitro expressed Akirin protein. GST-Twist pull-down of in vitro translated Daughterless provided as positive control for pull-down. Loading of GST-fusion proteins, as verified by Coomassie staining is provided. Note that (B) is a composite figure; lanes were omitted from the same gel for sake of clarity and direct comparison. doi:10.1371/journal.pgen.1002547.g002 

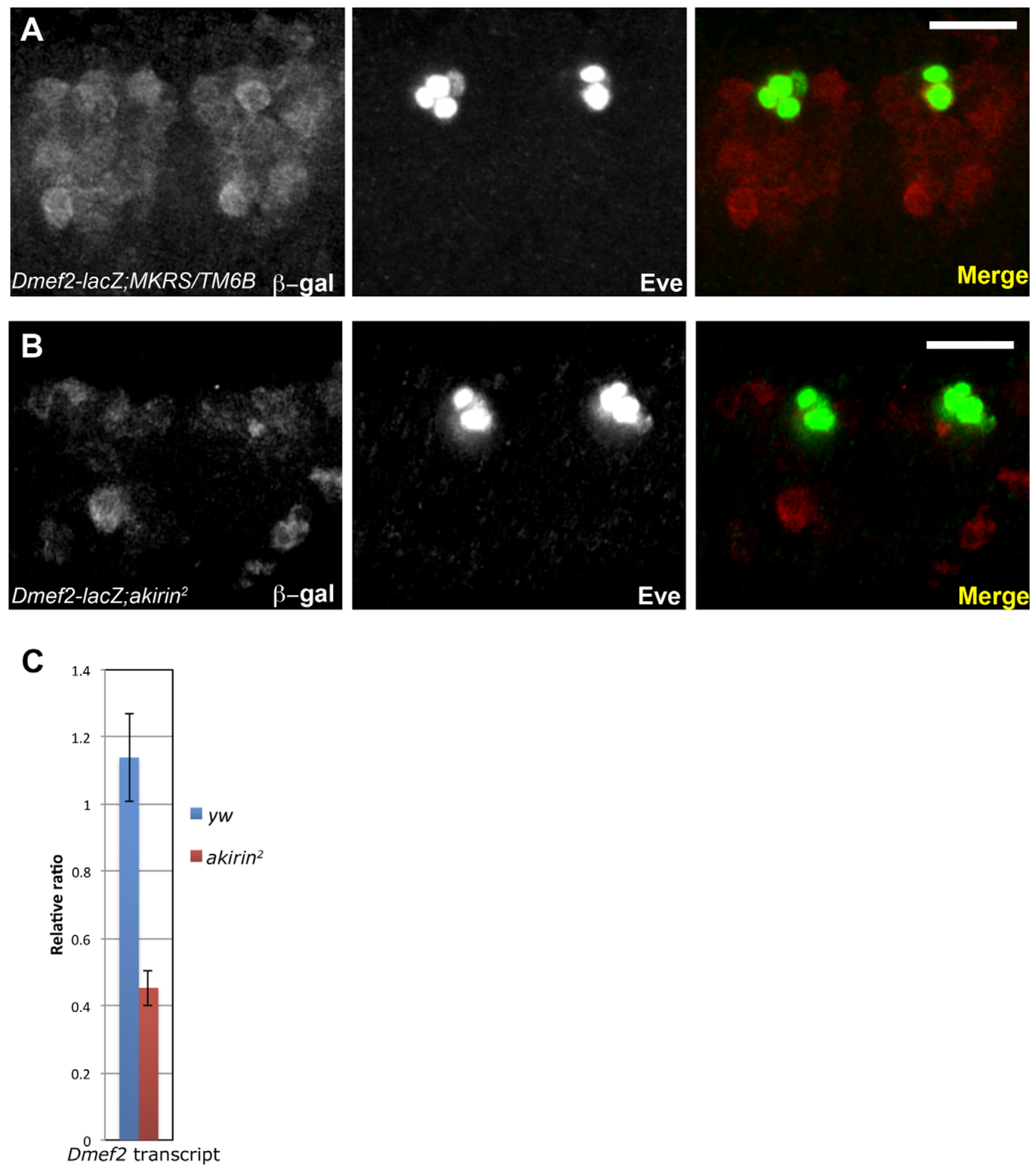

Figure 3. Dmef2 enhancer activity is reduced in akirin mutants. Confocal micrographs of two hemisegments of transgenic late stage 11 wildtype (A) and $a$ kirin $^{2}$ homozygous embryos (B) containing the Dmef2-lacZ reporter construct [33] showing immunoreactivity to $\beta$-galactosidase (red) and Even-skipped (Eve, green). Dmef2-lacZ reporter expression was reduced in akirin ${ }^{2}$ mutants compared to wild-type embryos. Images of wild-type and akirin $^{2}$ embryos were acquired with identical exposure settings (see Materials and Methods). (C) RT-PCR of Dmef2 mRNA isolated from 4-6 hourold wild-type and akirin ${ }^{2}$ mutant embryos demonstrates that Dmef2 expression is reduced in akirin ${ }^{2}$ mutants. Bar in (A) and (B) $=20 \mu \mathrm{m}$. doi:10.1371/journal.pgen.1002547.g003

modification that serves as a marker of actively transcribing loci in polytene chromosomes, was performed [51-53]. Akirin partially colocalized with Ser10-phosphohistone H3 staining $(43.8 \%$ colocalization, see Figure 4C, 4E and Figure S6A). To strengthen these results, we performed additional co-immunostaining for Akirin and Ser7-phosophorylated RNA polymerase II [54], which also showed partial colocalization between these two stains (55.4\%, see Figure 4D, 4F and Figure S6B). These data confirmed 

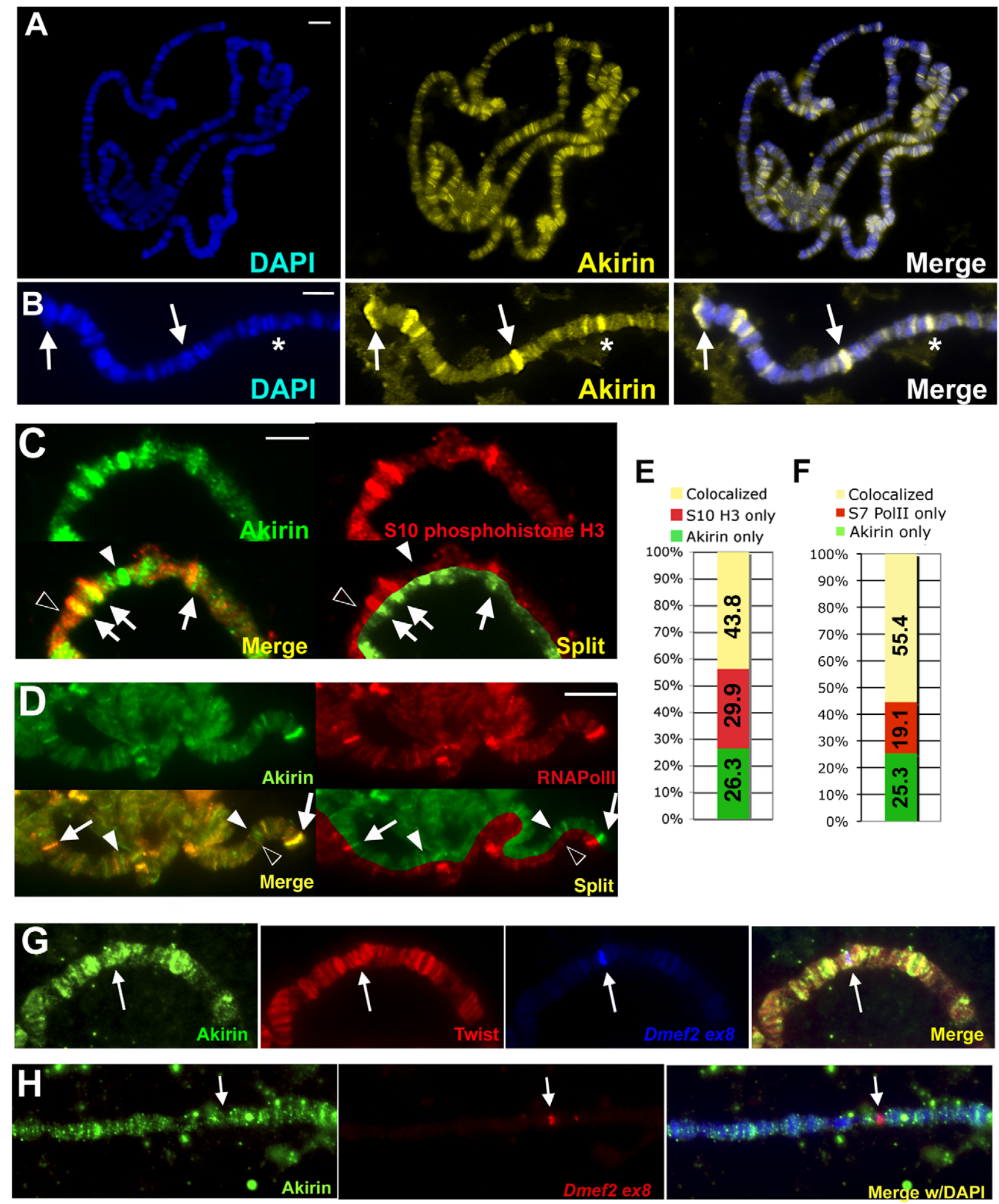

Figure 4. Akirin protein is broadly distributed throughout the genome. $(A, B)$ Polytene chromosomes were prepared from wild-type larvae, immunostained with antibody against Akirin and counterstained with DAPI to visualize DNA. Akirin is distributed at many sites throughout the genome and found at both puffed (arrows in (B)) and non-puffed (* in (B)) regions. (C,D) Detail of wild-type polytene chromosomes immunostained with antibodies against Akirin (green) and (C) Ser10-phosphorylated histone H3 (red) or (D) Ser7-phosphorylated RNA Polymerase II (red) indicate that Akirin is found at some but not all regions of active transcription in polytene chromosomes. See Figure S6 for whole-genome squashes. Split presentation provided to compare banding pattern. White arrows indicate colocalization, white arrowheads indicated Akirin-only bands, black arrowheads indicate Akirin-negative bands. (E,F) Quantification of colocalization between Akirin and (C) Ser10-phosphorylated histone H3 and (D) Ser7-phosphorylated RNA Polymerase II. (G) Twist and Akirin colocalize at Twist targets in polytene chromosomes. Chromosome squashes were 
prepared from larvae expressing Twist under the control of the Sgs3-GAL4 driver (Sgs3-GAL4>UAS-twist). Polytenes were hybridized with DNA probes against exon 8 of Dmef2, and further immunostained with antibodies against Akirin (green) and Twist (red). Both Twist and Akirin localize to the Dmef2 locus (arrow) when Twist is expressed in salivary glands. $(\mathrm{H})$ Akirin does not localize to the Dmef2 locus in salivary glands that do not express Twist. Scale bar in all images $=5 \mu \mathrm{m}$.

doi:10.1371/journal.pgen.1002547.g004

that Akirin is associated with some actively transcribing loci throughout the genome. In addition, because Twist is not expressed in wandering third instar salivary glands ([55] and data not shown), these data suggested that Akirin interacts with other transcriptional regulators for its function in this tissue. However, when we ectopically expressed Twist in the salivary glands using Sgs3-GAL4 [56], we found that Twist and Akirin partially colocalize on polytene chromosomes (54\% colocalization, Figure 4G). Furthermore, under these conditions both Twist and Akirin colocalized at the Dmef2 locus (Figure 4G), and Dmef2, which is not normally expressed in salivary glands, is now expressed (Figure S7). We note that Akirin is not localized to the Dmef2 locus when Twist is not present (Figure 4H). Together, these data supported our earlier results indicating that Akirin acts with Twist to promote the expression of a Twist-regulated target. Finally, the colocalization of Akirin with regions of active gene expression in a tissue that does not normally contain Twist confirmed that Akirin functions as a general regulator of gene expression.

\section{Akirin colocalizes with components of the Brahma chromatin remodeling complex}

One mechanism whereby Akirin might function as a general cofactor for gene expression would be through interactions with chromatin remodeling complexes. A Drosophila whole-genome yeast 2-hybrid experiment [57] suggested that Akirin interacts with BAP60, a core subunit of the Drosophila SWI/SNF class Brahma (BRM) chromatin remodeling complex [58]. Immunostaining of polytene chromosomes with antibodies against the Brahma (Figure 5A) and Snrl (Figure 5B) core subunits revealed that Akirin colocalized with BRM $(67 \%$ and $59.1 \%$ colocalization respectively, Figure 5D and Figures S8, S9). Akirin also colocalized with Osa $(65.3 \%$ colocalization), a subunit exclusive to the BAP complex (Figure 5C, 5D and Figure S9). These data therefore suggested that Akirin associates with BRM complex components but is not a core BRM complex subunit.

\section{Akirin interacts with BRM complex subunits during embryonic myogenesis}

Given the observed colocalization between Akirin and the Brahma, Snrl and Osa BAP subunits on polytene chromosomes, we hypothesized that there is a functional association of these proteins during embryonic development. To test this, the patterning of the somatic muscles in stage 16 embryos that are double heterozygotes for both akirin and brahma was examined (Figure 6B). Both brm $^{I 21} /+$ and akirin $^{2} /+$ (single heterozygous) embryos have a normal somatic muscle pattern (Figure S10), but the brm $^{I 21} /+$, akirin ${ }^{2} /+$ double heterozygous embryos (35\%, n = 55) displayed disruptions in the somatic muscles, with both missing and improperly attached muscles (Figure 6B, Table 2). We further analyzed embryos that are double heterozygotes of both akirin and other BRM complex core subunits (Figure 6, Table 2). Although heterozygous embryos for other BRM complex subunits do not show a somatic muscle phenotype (Figure S10), muscle phenotypes were observed in akirin $^{2} /+$, moira $^{l} /+(40 \%, \mathrm{n}=48$, Figure $6 \mathrm{C})$, akirin $^{2} /+$, Snrl $^{01319} /+\left(45 \%, \mathrm{n}=52\right.$, Figure 6D), and Bap60 ${ }^{1} /$ + ; akirin $^{3} /+$ embryos $(40 \%, \mathrm{n}=55$, Figure 6E). Additionally, akirin $^{2} /+,{\text { bap } 180^{486} /+(38 \%, \mathrm{n}=45 \text {, Figure 6F }) \text { and akirin }}^{2} /$
,+ osa ${ }^{2} /+(42 \%, \mathrm{n}=52$, Figure 6G) embryos also had disrupted muscle patterning. To ensure that these defects were specific for subunits of the BRM complex and not due to a general interaction between akirin and other chromatin factors, double heterozygous combinations of akirin with alleles of Polycomb, Nurf-38, Iswi, and $\mathrm{Su}(\mathrm{var}) 3-9$ were tested. Each of these allelic combinations showed muscle patterning defects in fewer than $5 \%$ of embryos. These results confirmed a functional interaction during muscle development between Akirin and core BRM subunits, as well as PBAP and BAP-specific subunits. Based on our data thus far, we hypothesized that Akirin mediates Twist-BRM interactions on a subset of Twist target genes. To begin to test this, we examined whether whether twi and brm genetically interact. We find that the somatic muscle pattern is disrupted in $\mathrm{twi}^{\mathrm{Il}} / \mathrm{+} ; \mathrm{brm}^{I 21} /+$ double heterozygous embryos $(23 \%, \mathrm{n}=51$, Figure $6 \mathrm{H})$, supporting a functional interaction between twist and brahma.

To determine whether the Akirin protein associates with the BRM complex in vivo, we attempted co-immunoprecipitation experiments from embryonic lysates using tagged Akirin. While we did observe weak physical interaction between tagged Akirin and the Brahma core subunit (data not shown), these appear to be highly transient and not robust. As we had identified a functional interaction between twist and brahma (Figure 6H), we examined whether Brahma and Twist physically interact. Antibodies against Brahma successfully co-immunoprecipitated Twist from whole embryonic extracts (Figure S11). Together these data suggest that Twist and Akirin both interact functionally with the BRM complex during myogenesis.

\section{Twist, Akirin, and a core BRM complex subunit are localized to Twist-dependent enhancers in vivo}

To further support our interaction data, we next tested whether Twist, Akirin, and the BRM complex were localized to Twistregulated enhancer elements in embryos when the muscle pattern is being established. Using chromatin immunoprecipitation and quantitative PGR, we examined the occupancy of the Dmef2 enhancer and the eve MHE element by Twist and Akirin (Figure 7). Both of these enhancer regions contain E-box elements that are bound by and regulated by Twist during development $[8,33,46,59]$. In agreement with previously published data [8], antibodies against Twist and Akirin both successfully immunoprecipitated the Dmef2 enhancer in extracts prepared from 2-4 and 4-6 hour-old embryos (Figure 7A, 7B). These data suggested that Akirin localizes to the Dmef2 enhancer element with Twist during these time periods. However, while Twist occupancy at the eve MHE was detected as previously reported [8], Akirin protein was not significantly enriched at the eve MHE region in 2-4, 4-6, and 6-10 hour embryo extracts compared to preimmune antisera controls. We concurrently examined occupancy of these elements by Twist and Akirin in extracts prepared from akirin ${ }^{2}$ mutant embryos. As we would predict, Twist occupancy at both Dmef2 and eve MHE enhancers was not significantly affected by the absence of Akirin (Figure 7A).

We next tested the occupancy of Dmef2 and eve MHE enhancers by a BRM subunit. We found that the BRM core subunit Moira occupied the Dmef2 enhancer early, in extracts prepared from 2 4 hour old embryos (Figure 7C), with a similar occupancy profile as that observed with Twist at this time. However, the reduction in 

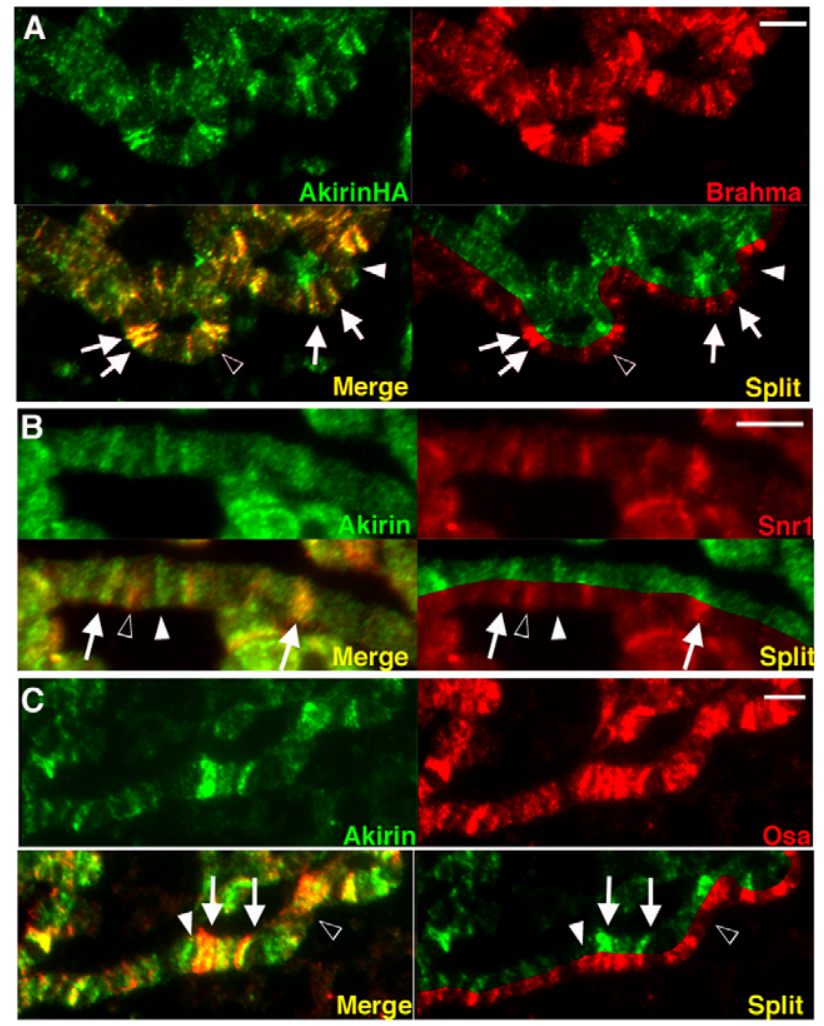

D
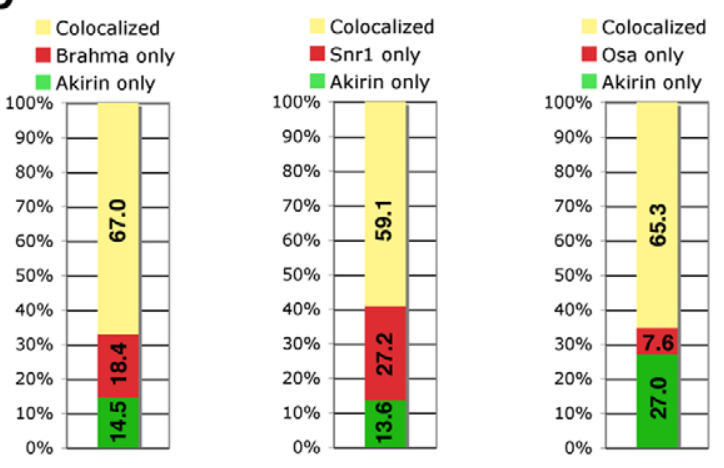

Figure 5. Akirin colocalizes with subunits of the SWI/SNF-class Brahma chromatin remodeling complex. (A-C) Detail of polytene chromosome squashes demonstrating immunocolocalization of Akirin with Brm complex subunits. See Figure S9 for whole genome squash images. (A) To avoid cross-reactivity between Akirin and Brahma primary antibodies, polytene chromosomes were prepared from larvae expressing HA-tagged Akirin (UAS-Akirin-HA) under control of the Sgs3GAL4 driver, and immunostained with antibodies against HA (green) and Brahma (red). Extensive colocalization was observed between Akirin-HA and Brahma (white arrows). Examples of Akirin-HA immunostain without Brahma colocalization (white arrowhead), and Brahma signal without Akirin colocalization (black arrowhead) are indicated. See Figure S8 for comparison of Akirin-HA and endogenous Akirin immunolocalization. $(B, C)$ wild-type polytene chromosomes were immunostained with antibodies against Akirin (green) and Snr1 (B) or Osa (C). Examples of Akirin signal without corresponding Snr or Osa colocalization are indicated (white arrowhead), and Snr or Osa signal without corresponding Akirin immunostain (black arrowhead) are indicated. (D) Quantification of colocalization/non-overlap between Akirin and Brm complex subunits -positive polytene bands in (A-C). Scale bar in all images $=5 \mu \mathrm{m}$.

doi:10.1371/journal.pgen.1002547.g005
Akirin level appeared to affect Moira occupancy at the Dmef2 enhancer, as we observed a $64 \%$ reduction in Moira levels at this enhancer in 2-4 hour old akirin $^{2}$ mutant extracts versus wild-type. We further found that Moira occupied the eve MHE, albeit at relatively low levels, at all time points examined. Further, this occupancy was not affected by the absence of Akirin, as the levels did not appear to change in akirin ${ }^{2}$ mutant extracts versus wildtype (Figure 7G). For comparison, we also examined occupancy of Twist, Akirin and Moira at the enhancer region of the oskar gene, which is not normally expressed in the embryo at this time [60]. We found no detectable occupancy of any of these factors at this region at the same timepoints (see Figure S12). In summary, we find that Twist, Akirin, and core subunits of the BRM complex all co-occupy the Dmef2 Twist-dependent enhancer together at a temporally critical time for establishing the muscle pattern during embryogenesis, and the occupancy of Moira at this element is dependent upon the presence of Akirin. In contrast, we find that Akirin is not present at a second Twist-regulated enhancer, the eve MHE, at the same period of embryonic development.

\section{Discussion}

The Twist transcription factor controls many key processes in the establishment and patterning of the mesoderm, including organogenesis of the somatic body wall musculature during Drosophila embryonic development [6]. As Twist activity regulates a large number of genes and processes over the course of mesodermal development $[8,9]$, specific Twist functions are likely conferred through secondary and tertiary proteins that interact with Twist [11]. In a screen for such interacting proteins, we identified the highly conserved nuclear protein Akirin. Akirin mutants show defects in myogenesis consistent with a role in regulating Twist activity, in particular at the Dmef2 gene. Akirin accomplishes this regulation through interactions with core subunits of the Brahma chromatin remodeling complex. Finally, we find Twist, Akirin and a BRM complex subunit all co-occupy the Dmef2 enhancer during early embryogenesis, and this occupancy of a core BRM subunit requires the presence of Akirin. These data suggest that Akirin is required for optimal expression of this Dmef2 enhancer element. By contrast, the Twistregulated eve MHE regulatory element does not require Akirin for its expression. Together, these results suggest that Akirin is an accessory protein that links Twist and the BRM chromatin remodeling complex for activation of specific Twist-regulated enhancers during Drosophila embryonic development. We propose that this Akirin-mediated link between Twist and the Brahma complex represents a novel paradigm for providing tissue and target specificity for Twist transcription factor activity with chromatin remodeling machinery during embryogenesis.

\section{Akirin and Twist interact during embryonic development}

Establishment of the somatic musculature during embryogenesis requires precisely regulated Twist activity [4]. The muscle phenotype of akirin mutants and twist and akirin double heterozygotes indicates a functional interaction between Twist and Akirin during myogenesis. This interaction is direct, as Akirin and Twist physically bind. Additionally, both Twist and Akirin cooccupy the Dmef2 enhancer during embryogenesis, and their association is important for robust expression from the Dmef2 enhancer. Dmef2 is a critical regulator of myogenesis and is expressed throughout this process [33,40,45,47,48]. Dmef2 coordinates multiple processes necessary for proper somatic myogenesis and regulates, in combination with Twist, a subset of Twist-regulated genes in a feed-forward manner [8,47]. The 

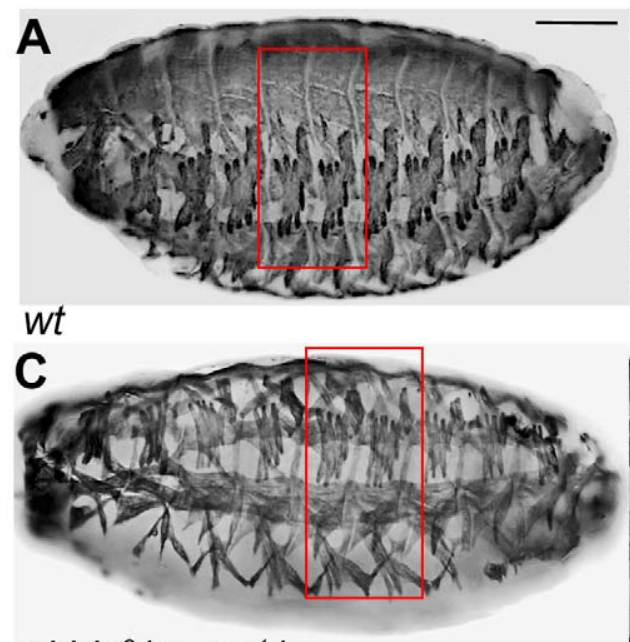

akirin $^{2} /+$, mor $^{1} /+$
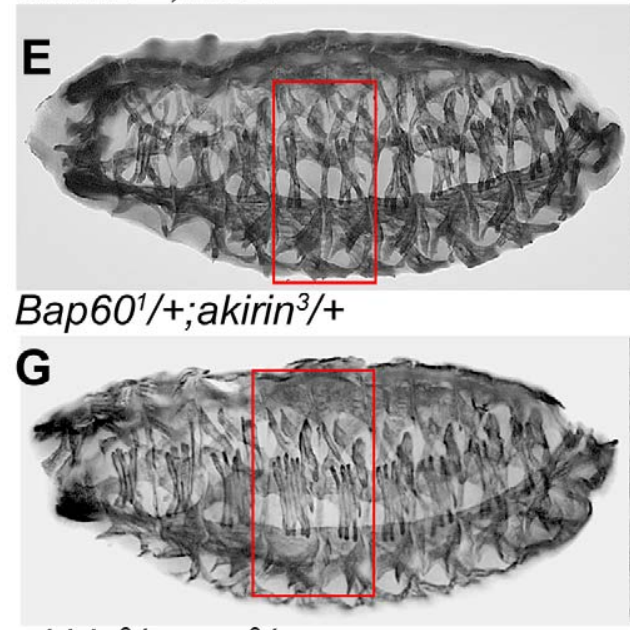

akirin $2 /+$, osa $^{2} /+$
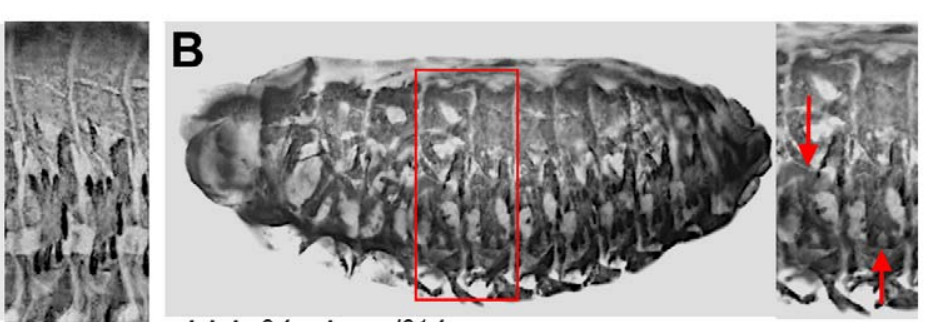

akirin $2 /+, b r m^{21 /+}$
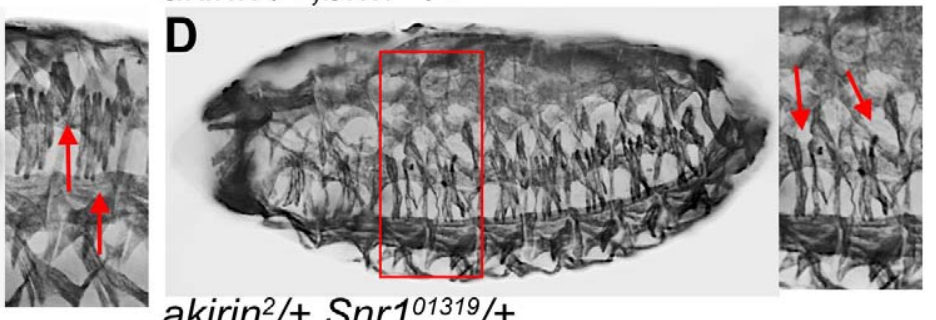

akirin $2 /+$, Snr101319/+
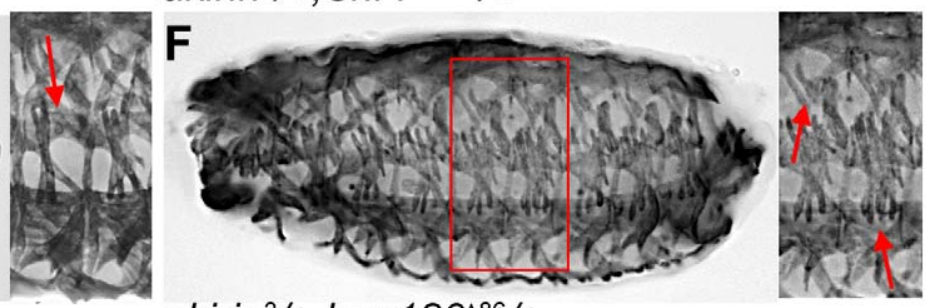

akirin $2 /+$, bap $180^{\wedge 86 /+}$
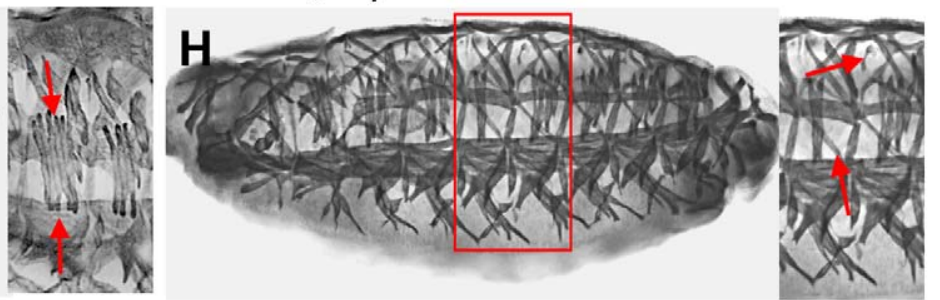

twilD96/+;brm ${ }^{121 /+}$

Figure 6. Akirin interacts genetically and physically with BAP and PBAP subunits during embryonic myogenesis. (A-G) Lateral views of stage 16 Wild-type $(w t)$ and embryos heterozygous for both akirin and indicated subunits of BAP and PBAP complexes. All embryos stained to reveal somatic musculature with tropomyosin antibodies. Thin red boxes in whole embryo photos indicate region shown in higher magnification for each embryo. Double heterozygous embryos have defects in general muscle pattering, suggesting a genetic interaction between akirin and components of the BRM chromatin remodeling complex (See Table 2 for quantification of defects). (H) Lateral view of stage 16 embryo heterozygous for both twist and brahma. Muscle patterning defects observed in these embryos suggest a genetic interaction between twist and brahma (A,C). Scale bar in panels $\mathrm{A}-\mathrm{H}=50 \mu \mathrm{m}$.

doi:10.1371/journal.pgen.1002547.g006

missing, misattached, or duplicated muscle phenotypes that we observe in akirin mutants are similar to the phenotypes of embryos in which Dmef2 or twist expression levels are modified [11,40-42]. For example, missing and misattached muscles were reported in incompletely rescued Dmef2 mutant embryos [41], while muscle duplications have been reported as a result of RNAi-mediated knockdown of twist [11]. Therefore, akirin mutant muscle phenotypes likely reflect a perturbation in Twist activity, resulting in an early alteration in Dmef2 expression levels during embryogenesis. There are other elements in the Dmef2 enhancer that are bound and controlled by transcription factors such as Pannier, Medea and Tinman, that regulate Dmef2 expression during embryogenesis $[8,33,46,59]$. It is unknown whether Akirin interacts with these factors at other Dmef2 control elements for their optimal expression or whether interaction with just one factor is sufficient. These questions are subjects for further study.

Whole genome ChIP-on-chip experiments have identified almost 500 cis-regulatory elements that are bound by Twist during mesodermal development [8]. Further, Twist co-regulates a number of targets together with Dorsal, Dmef2 and Tinman in a feed-forward manner. Interestingly, our data would argue that Twist does not require Akirin for optimal expression of all Twistdependent enhancers. First, our analysis of polytene chromosomes indicates that Akirin colocalizes with Twist at only $54 \%$ of Twist binding sites. Second, our chromatin immunoprecipitation experiments indicate that Akirin is enriched at the Dmef2 enhancer during the first 10 hours of embryonic development, but not at the even-skipped MHE enhancer at the same time. Accordingly, we did not observe a noticeable decrease in Eve expression, a reduction in Eve-positive clusters, or defects in patterning of the DA1 muscle in akirin mutants (Figure 3, Figure S3, and data not shown). What could explain the different requirements for Akirin for optimal expression of Dmef2 versus eve? On one level, there are inherent differences in the complexities of these enhancers: While both the eve MHE and Dmef2 enhancers contain E-boxes bound and regulated by Twist, 
Table 2. Genetic interactions between akirin and BRM complex subunit genes.

\begin{tabular}{|c|c|c|}
\hline Genotype & Embryos examined & $\%$ disrupted muscles ${ }^{a, b}$ \\
\hline twi $^{1} /+;$ akirin $^{2} /+$ & 60 & $21.6 \%$ \\
\hline $\operatorname{twi}^{1} /+;$ akirin $^{3} /+$ & 105 & $18.1 \%$ \\
\hline akirin $^{2} /+$, brm $^{121} /+$ & 55 & $35 \%$ \\
\hline $\operatorname{akirin}^{2} /+$, moira $^{1} /+$ & 48 & $40 \%$ \\
\hline akirin $^{2} /+$, Snr1 $01319 /+$ & 52 & $45 \%$ \\
\hline Bap $60^{1} /+;$ akirin $^{3} /+$ & 109 & $20.2 \%$ \\
\hline akirin $^{2} /+$, osa $a^{308} /+$ & 52 & $42 \%$ \\
\hline akirin ${ }^{2}$, bap $180^{486}$ & 45 & $38 \%$ \\
\hline$t w i^{1 /}+; b r m^{121} /+$ & 51 & $23 \%$ \\
\hline
\end{tabular}

the eve MHE element also contains multiple DNA elements bound by a large array of factors from the Wingless, Decapentaplegic, and RTK-Ras-MAPK pathways [59]. This greater complexity of regulation may obviate the need for Akirin to achieve optimal expression. On another level, the difference in Akirin regulation of these enhancers could be reflected in the local chromatin environment of these enhancers, and whether the individual enhancer needs to be remodeled by SWI/SNF activity for optimal expression. Analysis of deposited Modencode data [61] suggests that the histone modification profile of the eve MHE remains largely static during the same temporal window as our chromatin immunoprecipitation experiment, while the environment surrounding the Dmef2 enhancer element changes during this same time (data not shown). Further studies are required to identify the basis of this different requirement for Akirin at these Twist targets. Our polytene analysis indicates that a large number $(>200)$ of Akirin-positive bands colocalize with Twist in the genome (Figure 4). Identifying the complete list of Twist target genes that require Akirin will likely yield further clues as to the regulatory logic of Akirin together with chromatin remodeling during Twist target expression.

\section{Akirin interactions with transcription factors other than Twist}

Our data establishes Akirin as a Twist-interacting protein that promotes expression from a Twist-regulated enhancer; however, the results presented in this study also indicate that Akirin does not act solely with Twist.Analysis of salivary gland polytene chromosomes demonstrated that Akirin is associated with numerous actively transcribed gene loci. Twist is not normally expressed in salivary glands, therefore this result suggests that Akirin has roles in activation of non-Twist regulated genes. Moreover, the widespread expression of Akirin throughout the entire embryo suggests that specificity of Akirin function is determined not by restriction of Akirin expression, but rather by the associated transcription factor. Indeed, potential interactions between Akirin and other transcription factors have been described: Akirin misexpression enhances phenotypes resulting from mutations in the GATA-2 homologue pannier [62]. Additionally, whole genome yeast 2-hybrid analysis [57] suggests an interaction between Akirin and Charlatan, a zinc-finger transcription factor involved in development of the peripheral nervous system [63]. Finally, recent work has identified Akirin as a promyogenic factor and target for Myostatin regulation [30], as well as NF- $\kappa \mathrm{B}$ target gene expression in the Drosophila innate immunity pathway [29]. Taken together, these interactions with transcription factors other than Twist, and roles in non-Twist-dependent pathways further support our model whereby Akirin functions as a general transcription cofactor. We propose that the regulatory mechanism involving Akirin and the Brahma chromatin remodeling complex at specific enhancers is applicable to these transcriptional regulators in these other contexts. Further experimentation is required to validate this model.

\section{Akirin is likely a novel accessory of the Brahma chromatin remodeling complex}

Our studies identify Akirin as a nuclear factor that genetically interacts with the BRM complex and is required for optimal expression of the Twist-dependent Dmef2 enhancer. This association between Akirin and BRM complexes is likely the mechanism whereby Akirin is linked to gene activation. The Brahma complex (BRM) promotes gene activation by remodeling the local chromatin environment allowing components of the general transcription machinery greater accessibility to the DNA [15]. BRM complexes are tightly associated with regions of transcriptionally active chromatin, and are associated with both promoter paused (initiating) and actively elongating RNA Polymerase II complexes throughout the Drosophila genome [23]. Although a strong physical association of BRM complexes with RNA Polymerase II has not been confirmed, loss of BRM function leads to a severe impairment in transcription by RNA polymerase II [23]. Based on our genetic and ChIP data, we conclude from our results that Akirin is not a core BRM subunit, but is rather an accessory protein that is capable of interacting with BRM complexes. We base this conclusion on the observation that the distribution of Akirin and BRM subunits on polytene chromosomes do not completely overlap, and because numerous biochemical analyses of BRM complex composition to date have failed to identify Akirin as a BRM subunit $[24,64]$. Further, we did not observe a specific interaction of akirin with either BAP or PBAP complex-specific subunits during myogenesis (Figure 6). In Drosophila, both BAP and PBAP complexes have both been linked to gene activation and repression, are present in the same cells, and perform unique, yet cooperative, functions during development [15,16,19,24-28]. Indeed, while we were able to observe weak physical interactions between Akirin and the Brahma core subunit in vivo (data not shown), these interactions were not overly robust. Moreover, it is unknown if Akirin needs to be post-translationally modified or to further associate with other factors to mediate a physical interaction with the Brahma subunit. Further, while we tested interactions with the core Brahma subunit, it remains to be determined whether Akirin may be interacting instead with other core BRM subunits. Nevertheless, our data strongly suggests a likely association as an accessory of the BRM complex. As an accessory protein, Akirin would likely confer tissue, target, and even temporal specificity on BRM complex activity by connecting BRM complexes with a particular transcription factor for promotion of gene expression (see below).

\section{Interactions among Akirin, BRM complexes, and Twist}

Our data suggest that Twist target genes have different requirements for the presence of chromatin remodeling factors during gene activation and imply that the chromatin environments at these genes are varied. This also would suggest that the local 


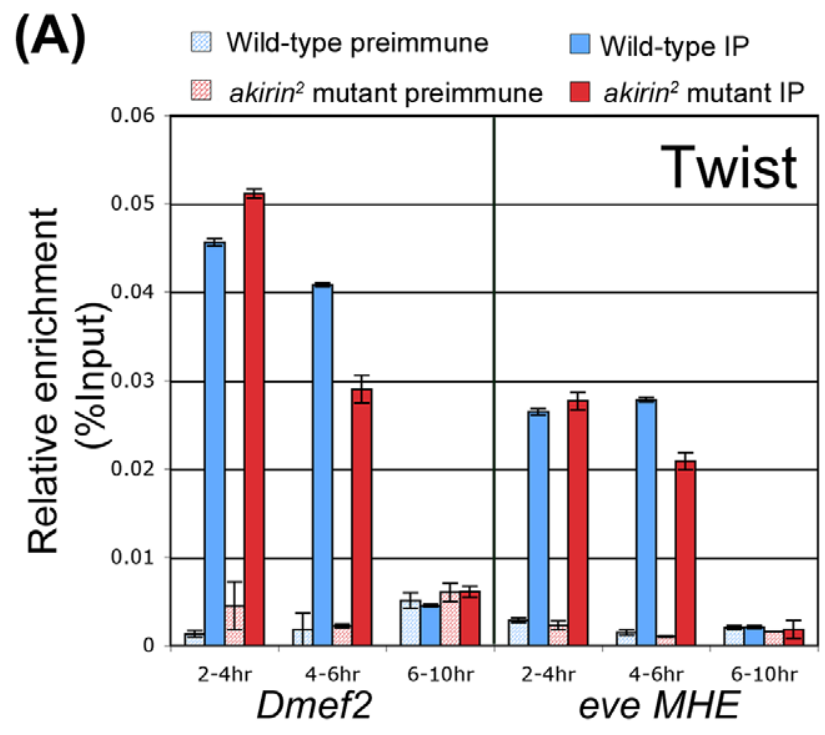

(B)
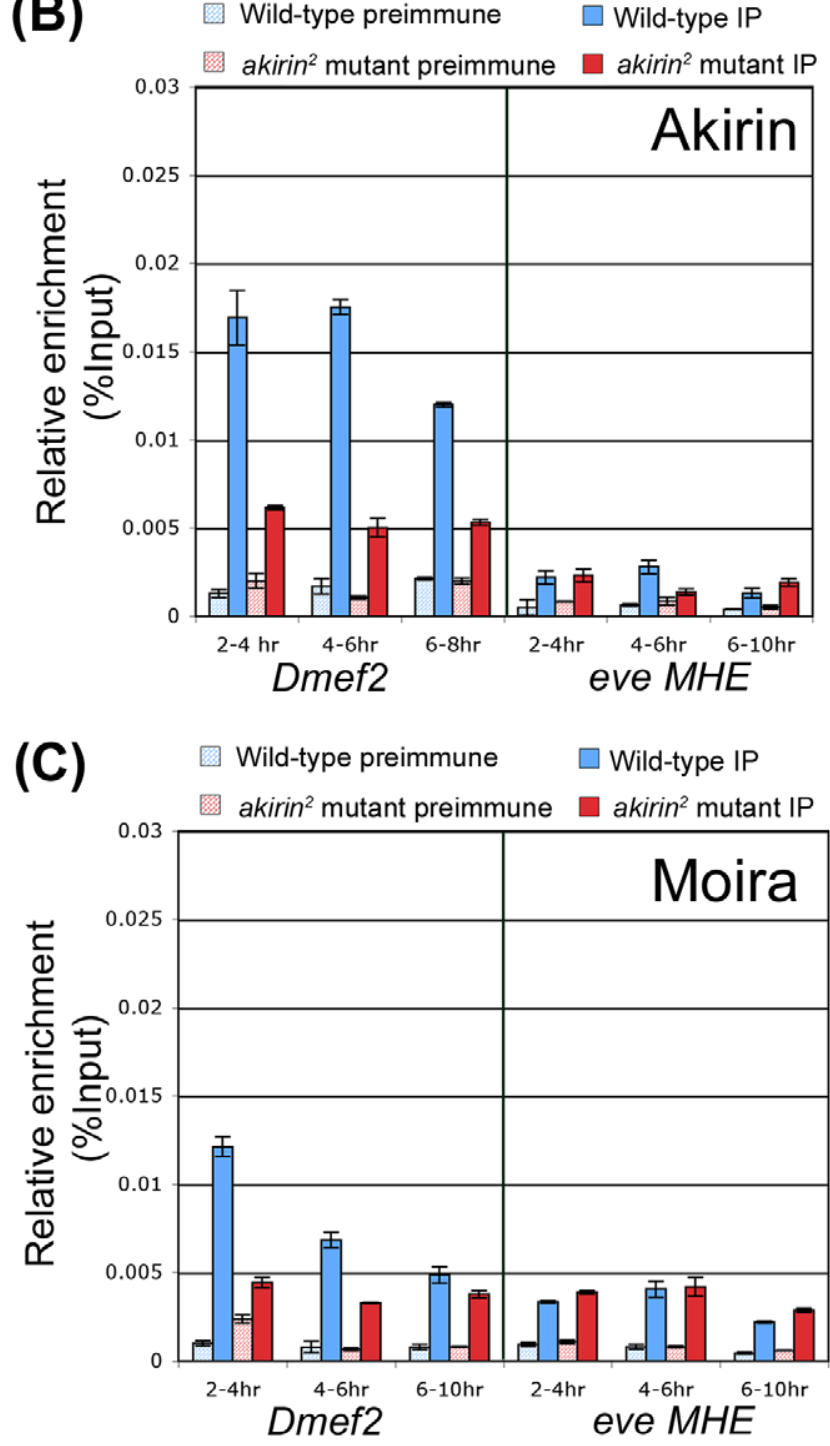

Figure 7. Akirin and the BRM complex co-occupy the Dmef2 enhancer during embryogenesis. Chromatin immunoprecipitations were performed on extracts prepared from $2-4,4-6$, and 6-10 hour-old yw (Blue bars) and akirin $^{2}$ mutant (Red bars) embryos using anti-Twist (A), anti-Akirin (B), and anti-Moira (C) antibodies. The presence of the Dmef2 enhancer or even-skipped MHE enhancer (eve MHE) was assayed in precipitated DNA samples using quantitative PCR. Values for preimmune (stippled bars) and immunoprecipated samples (solid color bars) are presented as percent of total input fraction. Akirin and the core BRM subunit Moira both co-occupy the Dmef2 enhancer with Twist in 2-4 and 4-6 hour-old embryos. The occupancy of Moira at this enhancer appears to be Akirin-dependent, as Moira occupancy decreases by approximately $64 \%$ in akirin mutant extracts. (A-C) While Twist and Moira occupy the eve MHE enhancer, Akirin is not enriched at this region in wild-type or mutant extracts, and the absence of Akirin does not affect the occupancies of either Twist or Moira. doi:10.1371/journal.pgen.1002547.g007

chromatin environment of a particular Twist target changes over developmental time. Further experiments will be required to validate this hypothesis. As an accessory protein, Akirin optimizes Twist transcription factor activity outputs. Akirin likely accomplishes this optimization by facilitating an interaction between Twist and BRM complexes and as such, we would predict, change the local chromatin environment to one more favorable for transcription. The exact nature of the interface between bHLH transcription factors such as Twist and chromatin remodeling complexes such as BRM has not been previously determined. Our data would suggest that Akirin would be a suitable candidate for mediating this relationship between Twist and chromatin remodeling complexes. Mammalian SWI/SNF complexes are positively associated with bHLH transcription factor activity; however, the precise role of their remodeling activity during expression of bHLH target genes remains unclear [65]. Whether a similar linkage via Akirin is at play with mammalian Twist during development or in a cancer context remains to be tested. Nevertheless, in keeping with our proposed model of Akirin function, our data suggest a relationship between Twist and BRM during development: $t w i i^{I I} /+; b r m^{2} /+$ double heterozygous embryos show muscle patterning defects similar to wwi $^{1} /+$;akirin ${ }^{2} /+$ double heterozygotes (Figure 6). Also, forced expression of Twist in salivary glands and subsequent analysis of colocalization on polytene chromosomes indicated that Twist and Brahma colocalized $58 \%$ of the time (Figure S11), a frequency similar to that observed between Twist and Akirin (54\%, Figure 4 and data not shown).

Our finding that early (i.e., 2-4 hours) occupancy of the Moira core subunit at the Dmef2 enhancer was decreased in akirin mutants would suggest that Akirin contributes to BRM complex localization. However, our co-immunoprecipitation experiments (data not shown) would suggest that any physical interaction between these two proteins would be either highly transient, or exquisitely sensitive to the presence of interfering factors such as protein tags. Therefore, the mechanism by which Akirin would increase Moira occupancy remains unclear. The result of such a recruitment or stabilization of BRM complexes by Akirin at Twist-target loci, would presumably result in remodeling of the local environment by BRM for optimal gene expression. Further experiments, aimed at understanding the nature of the Akirin/BRM complex association are currently underway. Together, this association between Twist, Akirin and the BRM complex would provide a novel mechanism linking chromatin remodeling factors to spatiotemporal-specific gene activation by the Twist transcription factor. Our work provides another venue to investigate how changes in the chromatin environment at specific targets leads to optimal gene expression and how these local changes impact the development of specific tissues. 


\section{Materials and Methods}

\section{Drosophila genetics}

Flies were reared using standard conditions. Three different alleles of akirin were used: akirin ${ }^{\text {KG01343 }}$ (referred to in this work as

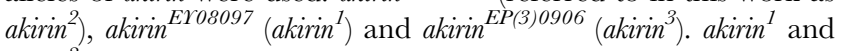
akirin $^{2}$ are viable P-element insertions into the first intron of akirin, while the homozygous lethal akirin ${ }^{3}$ allele is an insertion into the first exon (Figure 1A). We further generated two additional homozygous lethal akirin alleles by excision of the akirin ${ }^{1}$ (EY08097) P-element (akirin ${ }^{4}$ and akirin $\left.^{5}\right)$. Mobilization of the Ero8097 Pelement resulted in the isolation of two different mutations: akirin ${ }^{5}$, which is an insertion of approximately $1 \mathrm{~kb}$ of DNA into the second akirin exon due to incomplete P-element excision, and akirin $^{4}$, which is a deletion of $6 \mathrm{~kb}$ containing the first exon and intron of akirin, as well as a portion of the upstream $S H 3 \beta$ open reading frame. The akirin ${ }^{E P(3) 0906}$ mutant chromosome possessed a second unidentified lethal mutation in addition to the EP(3)0906 insertion. This second lethal mutation was removed by recombination with a rucuca-marked chromosome [66] to produce the akirin $^{3}$ line used in this study. akirin ${ }^{3}$, akirin ${ }^{4}$, and akirin ${ }^{5}$ homozygous mutant embryos die shortly after stage 16 and do not hatch into larvae (data not shown). For analysis, akirin ${ }^{3}$, akirin ${ }^{4}$, and akirin $^{5}$ were balanced over TM3, Dfd-lacZ or TTG [67]. For genetic interactions, the following alleles were used: moira ${ }^{1}$, Snr1 ${ }^{01319}$, brm $^{2}$ and brm $^{I 21}$ (Bloomington stock collection). osa ${ }^{308}$ and $b a p 180^{486}$ (a gift of J. Treisman), Bap60 (a gift of G. Mardon), nurf ${ }^{38}, I_{s w i}{ }^{1}$, and Su(var)3-9 (a gift of V. Corces), and twi ${ }^{1}$ [39]. Dmef2-lacZ [33] and eveMHE-lacZ (a gift of A. Michelson) were used for expression studies. GAL4 expression lines [68] used were twi-GAL4;Dmef2-GAL4 [69] and Sgs3-GAL4 [56]. OreR and yw flies were used as wild-type stocks where indicated. Germline clones [37] were generated by heat shock of $h s-F L P$; ovo $D^{I}, F R T 2 A$ / akirin $^{3}$,FRT2A larvae. Germline clone females were then mated to akirin $^{3} / T M 3, D f d$-lacz at $20-22^{\circ} \mathrm{C}$ to create akirin ${ }^{3}$ maternal/ zygotic embryos.

\section{Production of UAS-HA-tagged Akirin flies}

The HA-tag was fused in-frame at the Akirin C-terminus using PCR techniques (Table 3). PCR products were cloned into pUASt and injected into yw embryos as described [4]. Multiple independent transformant lines were generated and evaluated for Akirin-HA expression.

\section{Double-interaction screen}

Briefly, we adapted the method described by L. Pick and colleagues [31]. Enhancers tested were the 175-bp upstream enhancer of Dmef2 [33] and the 375-bp enhancer contained in the first intron of the tinman gene [34] cloned upstream of a HIS3 reporter. Reporter strains were created using these constructs. Screening for Twist-interacting proteins was performed by introducing a cDNA library prepared from 0-6 hour Drosophila embryos fused to the GAL4 activation domain [31]. Akirin was identified as a cDNA that expressed the HIS3 reporter in a Twistdependent manner at both Dmef2 and tinman enhancers [32].

\section{Immunohistochemistry and production of Akirin antibody}

Antibodies and dilutions used: anti-tropomyosin (1:1000, Abcam), anti-myosin monoclonal antibody (1:500, a gift of $\mathrm{S}$. Abmayr), anti-Twist (1:100, a gift of M.Levine) anti-Osa (1:50, DSHB), anti-Brm (1:50, gift of C.P. Verrijzer), anti-HA 3F10 (1:25, Roche), anti-Dmef2 (1:1000, a gift of B. Patterson), antiSnr 1 (1:100, gift of A. Dingwall), anti-Eve (1:3000, a gift of M. Frasch), anti-phosphohistone H3 (mouse monoclonal, 1:100, Millipore), anti-Serine-7-phosphorylated RNA polymerase II (1:100, Millipore) and anti- $\beta$-galactosidase (1:1000, Abcam). Whole-mount embryo immunohistochemistry was performed as described [70]. Comparison of $\beta$-galactosidase expression levels in wild-type and akirin mutant embryos was performed as per [11]. For production of polyclonal Akirin antibody, the peptide sequence of CESMIKERENQLR corresponding to residues 151-163 in full-length Drosophila Akirin was synthesized and

Table 3. Primers used in this study.

\begin{tabular}{|c|c|}
\hline Sequence & Purpose \\
\hline 5'-CGCCTCGAGGCTTAGCCAGCGTAGTCTGGGACGTCGTATGGGTACGACAGGTAGCTAGGCGCTGC- 3' & Upstream primer for HA-tagging of Akirin \\
\hline 5'-CTCCCCCGTCTCCATAAAGGTC-3' & Upstream primer- production of Dmef2 ISH probe \\
\hline 5'-GTTGCTACTGGTGCTGCTGCTG-3' & Downstream primer- production of Dmef2 ISH probe \\
\hline 5'-AACTGCCAAGCGTGTGCCGTGT-3' & qPCR detection of Dmef2 transcript- upstream primer \\
\hline 5'-CAAGGCCAAAGGGGCAGCACCA-3 & qPCR detection of Dmef2 transcript- downstream primer \\
\hline 5'-CTTCTTCAGCGACACCCATT-3' & qPCR detection of GAPDH transcript- upstream primer \\
\hline 5'-ACCGAACTCGTTGTCGTACC-3' & qPCR detection of GAPDH transcript- downstream primer \\
\hline 5'-CCGATGCTGCTGCTGCTGCTACT-3' & Forward qPCR primer used in ChIP of Dmef2 enhancer (-2344) \\
\hline 5'-GACCATGTACCCCGATGCTGTGC-3' & Reverse qPCR primer used in ChIP of Dmef2 enhancer $(-2257)$ \\
\hline 5'- CTCCCCCGTCTCCATAAAGGTC-3' & Forward qPCR primer used in ChIP of Dmef2 ORF $(+11007)$ \\
\hline 5'- GTTGCTACTGGTGCTGCTGCTG-3' & Reverse qPCR primer used in ChIP of Dmef2 ORF $(+10782)$ \\
\hline 5'- GGAAATCGTCTTGGGATGCGAGTGGT-3' & Forward qPCR primer used in ChIP of eve MHE region (+6171) \\
\hline 5'- AGCTGCAGATCCGGACTCGCAATAG-3' & Reverse qPCR primer used in eve MHE region (+6334) \\
\hline 5'- CTGGGTCGCTTGGAGAAGGAGTT-3' & Forward qPCR primer used in ChIP of eve ORF $(+490)$ \\
\hline 5'- CGATCCTCTGACGCTTGTCCTTC-3' & Reverse qPCR primer used in eve ORF $(+616)$ \\
\hline 5'-TGCGAATGGTCTTCATGGAA-3' & Forward qPCR primer used in ChIP of oskar enhancer (+8117) \\
\hline 5'-CACCGTCAAGCAGCGTGTAC-3' & Reverse qPCR primer used in ChIP of oskar enhancer (+8182) \\
\hline
\end{tabular}


injected into rabbits (Sigma). Production bleeds were tested for immunoreactivity using pre-immune serum as a negative control (See Figure S2).

\section{Microscopy}

Bright field and immunofluorescent images were obtained using a Zeiss Axiophot microscope. Images were processed using Adobe Photoshop. Confocal images were acquired using either a Zeiss LSM 510 confocal scanning system mounted on an Axiovert $100 \mathrm{M}$ microscope equipped with a $63 \times 1.2 \mathrm{NA}$ C-Apochromat water objective, or a Leica SP5 confocal microscope equipped with a $63 \times 1.4$ NA HCX PL Apochromat oil objective. Pinholes were set to capture optical slices of $1.0 \mu \mathrm{m}$. All images were processed using Adobe Photoshop. Maximum intensity projections of confocal Z-stacks were rendered using Volocity Visualization (Improvision).

\section{GST-pulldown assays}

Twist was cloned into pGEX-2T in frame and fusion protein expressed via IPTG induction. Glutathione Sepharose 4B beads (Amersham Pharmacia Biotech) bound with GST or GST-Twist were suspended in ZTx buffer (25 mM HEPES, $\mathrm{pH}=7.5$, $12.5 \mathrm{mM} \mathrm{MgCl} 2,20 \%$ glycerol, $0.1 \%$ Triton-X 100, $150 \mathrm{mM}$ $\mathrm{KCl})+0.25 \mathrm{mg} / \mathrm{ml} \mathrm{BSA}$ and $1 \mathrm{mM}$ DTT and incubated for 10 mins with rotation. ${ }^{35} \mathrm{~S}-$ Methionine-labeled Akirin and Daughterless proteins were produced in vitro using the TnT coupled reticulocyte lysate system (Promega). Beads and translated proteins were then incubated at room temperature with rotation for 1 hour. Beads were spun and washed in NETTx buffer $(20 \mathrm{mM}$ Tris, $100 \mathrm{mM} \mathrm{NaCl}, 1 \mathrm{mM}$ EDTA and $0.25 \%$ Triton-X 100). Proteins were eluted from beads by boiling in Laemmli sample buffer and resolved by SDS-PAGE. Gels were fixed in $50 \%$ Methanol, 10\% Acetic Acid, incubated in Amplify solution (Amersham Pharmacia Biotech), and dried for autoradiography.

\section{Western blotting}

Whole-embryo extracts were prepared and resolved by SDSPAGE as described [71]. Western detection was performed as described [72]. Antibodies and dilutions used: anti-Dmef2 (rabbit, 1:1000) (a gift of B. Patterson), anti-alpha-tubulin (mouse, 1:5000) (Sigma), anti-Brahma (1:1000) (a gift of C.P.Verrijzer), anti- $\beta$ galactosidase (mouse, 1:1000) (Promega).

\section{Densitometry}

Autoradiographs were scanned using a BioRad GS-800 Calibrated Densitometer to determine optical density of each band, which was then divided by the area examined. For analysis of $\beta$-galactosidase levels (see Figure S5), this value was normalized to similar results obtained from anti- $\alpha$-tubulin loading controls. For analysis of co-immunoprecipitations (see Figure 6), this value was reported as a percentage of the input sample analyzed. For each condition, background (chosen from blank region of the film piece, with no sample present) was subtracted from all analyzed regions prior to analysis.

\section{Polytene chromosome immunohistochemistry, in situ hybridization, and immunocolocalization analysis}

Polytene squash preparations and immunostaining was performed as described [52]. For colocalization analysis, separate channel information was compared for signal bands in polytene squashes. Contrast settings were set to maximum for each channel, eliminating $>80 \%$ of the background signal. A band was scored as "colocalized" when it appeared in both channels. Bands that appeared solely in either signal channel were scored accordingly. A minimum of 150 bands were counted from at least three different squash preparations. Data was plotted in percentage bar graphs using Microsoft Excel.

In situ probes were generated using the Roche DIG-labeled PCR kit, using oligonucleotides to amplify exon 8 of the Dmef2 open reading frame (Table 3). In situ probe purification and hybridization was performed as described [73]. Following hybridization, slides were washed in increasing stringency SSC solutions, and then incubated in polytene buffer. Antibody staining was carried out as described above, using 1:100 rhodamineconjugated anti-DIG antibodies (Roche) to detect DIG-labeled probes.

\section{Production of CDNA and quantitative PCR}

wild-type (yw) and akirin ${ }^{2}$ embryos were collected on $10 \mathrm{~cm}$ apple juice agar plates and allowed to grow to $4-6$ hours of age after egg laying. Embryos were dechorionated in bleach and homogenized in RNA TriReagent (Sigma). Total mRNA was prepared according to manufacturer's instructions, and resuspended in RNAse-free water. Following purification, total mRNA was treated with DNAseI to degrade genomic DNA, and repurified using phenol:chloroform. 1 microgram of total mRNA was used to prepare first-strand cDNA using the RevertAid First Strand Synthesis Kit (Fermentas). First-strand product was diluted to produce a working concentration of $10 \mathrm{ng} / \mu \mathrm{L}$. For quantitative PCR, first-strand cDNA product was diluted 1:2 with Lightcycler 480 SYBR green master mix (Roche). qPCR was conducted using a Lightcycler 480 qPGR machine (Table 3). qPCR results were analyzed and relative ratios of Dmef2 transcripts (normalized to GAPDH levels) in yw and akirin ${ }^{2}$ mutants were determined using described methods [74].

\section{Co-immunoprecipitations}

Total nondenatured extracts were prepared from yw embryos collected on apple juice plates. To prepare total nondenatured extracts, embryos were dechorinated in $50 \%$ bleach, rinsed in distilled water, and immediately homogenized in one $\mathrm{mL}$ of extraction buffer (50 mM HEPES, pH 7.6, $385 \mathrm{mM} \mathrm{NaCL}, 0.1 \%$ Tween-20, $0.1 \mathrm{mM}$ EGTA, $1.1 \mathrm{mM} \mathrm{MgCl}_{2}$ and $100 \mu \mathrm{g} / \mathrm{mL}$ PMSF, and $1 \mu \mathrm{g} / \mathrm{mL}$ each of Aprotinin, leupeptin, and pepstatin A), using a $1 \mathrm{~mL}$ Dounce homogenizer. Extracts were spun at $15,000 \mathrm{~g}$ at $4^{\circ} \mathrm{C}$ to pellet debris. Supernatants were removed and following the addition of glycerol to $10 \%$, extracts were snapfrozen in liquid nitrogen before storing at $-80^{\circ} \mathrm{C}$ for later use. For immunoprecipitations, extracts were thawed and quantitated using the BCA assay method (Pierce). Extracts were diluted in IP buffer (10 mM HEPES pH 8, $100 \mathrm{mM} \mathrm{NaCl,} \mathrm{10 \%} \mathrm{glycerol,} 0.05 \%$ Tween-20, $100 \mu \mathrm{g} / \mathrm{mL}$ PMSF, and $1 \mu \mathrm{g} / \mathrm{mL}$ each of Aprotinin, leupeptin, and pepstatin A) to produce a final concentration of $3 \mathrm{mg} / \mathrm{mL}$ of total protein. Extracts were incubated with $10 \mu \mathrm{L}$ of anti-Brahma antibody (a gift of P. Verrijzer) for 1 hour at $4^{\circ} \mathrm{C}$. Immunocomplexes were pulled-down using $20 \mu \mathrm{L}$ of a $50 \%$ protein A-agarose slurry. Beads were washed four times at $4^{\circ} \mathrm{C}$, and immunocomplexes were released by boiling in Laemmli sample buffer.

\section{Chromatin immunoprecipitation (ChIP)}

Chromatin immunoprecipitations were performed essentially as described [75] with several modifications. In brief, 2-4 hour, 4 6 hour, 6-10 hour yw Drosophila embryos were collected, dechorionated, and fixed in fixing solution (1 mM EDTA, $0.5 \mathrm{mM}$ EGTA, $100 \mathrm{mM} \mathrm{NaCl}, 2 \%$ formaldehyde (v/v), $50 \mathrm{mM}$ HEPES, $\mathrm{pH}$ 8.0) with an equal volume of $\mathrm{n}$-heptane by vigorous shaking 
for $25 \mathrm{~min}$ at room temperature. Fixed embryos were washed twice with methanol, once with storage buffer $(50 \mathrm{mM}$ Tris-HCl $\mathrm{pH}$ 8.0, $1 \mathrm{mM}$ EDTA), and kept at $-80^{\circ} \mathrm{C}$. Approximately $100 \mu \mathrm{L}$ of fixed embryos were washed with $1 \mathrm{ml}$ of IP buffer (100 mM NaCl, $67 \mathrm{mM}$ Tris pH 8.0, 0.33\% SDS, 1.66\% Triton $\mathrm{X}-100,5 \mathrm{mM}$ EDTA) for $10 \mathrm{~min}$. The following procedures were all carried out at $4^{\circ} \mathrm{C}$. Embryos were resuspended in IP buffer containing protease inhibitor cocktail, were then sonicated fourteen times for $12 \mathrm{sec}$ by a Branson 250 sonicator at a power setting of 4 and $30 \%$ duty cycle. After sonication, the Whole Cell extract; (WCE) was obtained by centrifugation. $30 \mu \mathrm{L}$ of WCE was diluted, reverse cross-linked, and treated with proteinase $\mathrm{K}$. DNA was purified using QIAprep Spin Miniprep columns and recovered in $100 \mu \mathrm{L}$ of elution buffer (Qiagen) to check genomic DNA fragments with an approximate bulk size of 300-800 bp. For each immunoprecipitation, WCE containing $75 \mu \mathrm{g}$ chromatin DNA was treated with either $5 \mu \mathrm{L}$ of nonimmune guinea pig serum, anti-Twist antiserum (guinea pig), anti-Akirin antiserum (rabbit), and anti-Moira antiserum (rabbit), together with $25 \mu \mathrm{L}$ of protein A agarose coated with salmon sperm DNA (Upstate) for overnight incubation. Mock immunoprecipitations were performed using nonimmune guinea pig serum, preimmune rabbit serum, and nonimmune rabbit serum. Precipitated agarose beads were washed with mixed micelle buffer, buffer 500, LiCl/ Detergent buffer, and TE, then treated with RNase A. The precipitated immune complexes were eluted by Bicarbonate/SDS buffer and cross-links were reversed in the presence of proteinase $\mathrm{K}$ overnight at $65^{\circ} \mathrm{C}$. DNA was purified using QIAprep Spin Miniprep columns and recovered in $200 \mu \mathrm{L}$ of elution buffer. Quantitative PCR was performed using the Applied Biosystems (ABI) Prism 7700 Real-Time qPCR instrument (AQ method). One of triplicates of reaction mixture were prepared by adding 4 ul $2 \times$ SYBR mix (usb 75762), 0.4 $\mu \mathrm{L} 2.5 \mathrm{uM}$ Primers (Table 3), and $3.6 \mu \mathrm{L}$ DNA template. To depict standard curves, duplicates of each 5, 50, and 500 folds diluted DNA from WCE as described above were used. \%input and SD were calculated from triplicated scores of immunoprecipitations over that of input WCE.

\section{Supporting Information}

Figure S1 Specificity of Akirin antibody. (A) Pre-immune serum and anti-Akirin immunoserum were used to stain duplicate Western blots containing wild-type whole-embryo extracts. Volume of extract loaded is indicated above in microliters. AntiAkirin immunosera detects a double band of approximately 22 and $20 \mathrm{kDa}$. The same doublet is not detected with pre-immune serum at identical concentration. Anti- $\boldsymbol{\alpha}$-tubulin included as a loading control. (B) Akirin is significantly decreased in akirin ${ }^{3}$ mutant extracts. Twenty-five (25) akirin ${ }^{3}$ homozygous mutant and akirin $^{3} /+6-10$ hr-old heterozygote embryos were loaded in each lane, and stained with anti-Akirin antibodies. (C) Polytene chromosomes were prepared from OregonR larvae and stained with preimmune serum or anti-Akirin immunoserum as indicated, at identical concentrations. Images were acquired with identical gain and acquisition settings. Scale bar $=5 \mu \mathrm{m}$.

(TIF)

Figure S2 Akirin protein is expressed broadly throughout the embryo and is localized to the nucleus. (A) Hemisegment of wildtype embryo immunostained with antibodies against Akirin and Lamin, which labels the nuclear envelope. Scale bar $=20 \mu \mathrm{m}$. (B) twi-GAL4;Dmef2-GAL4>UAS-Akirin-HA embryos stained with antibodies against HA and Twist. HA-tagged Akirin localizes to the nucleus with Twist in these embryos. Scale bar $=20 \mu \mathrm{m}$. (TIF)
Figure S3 akirin mutant embryos display a range of mutant muscle phenotypes. (A) Genomic map of akirin locus, showing location of P-element insertions and corresponding akirin mutant alleles used in this study. (B) Whole embryo presentations of akirin mutant muscle phenotypes. Lateral views of stage 16 wildtype (i, wt) and akirin mutant (ii, iii, iv) embryos demonstrate the types of muscle phenotypes observed. All embryos stained with anti-tropomyosin to reveal somatic musculature. All allelic combinations are listed as maternal/paternal contribution. For clarity, the LT muscles are used to illustrate the following predominant muscle defects observed in akirin mutants (red arrows): (ii) improperly attached muscles, (iii) duplicated muscles, and (iv) missing muscles. In all figures, anterior is to left and dorsal is up. Scale bar $=50 \mu \mathrm{m}$. (C) akirin mRNA is maternally loaded. RT-PGR for akirin and twist mRNA performed using total mRNA isolated from 0-1 hour wild type embryos. Plasmid controls provided as positive amplification controls. rp49 amplification included as positive control for a maternally deposited message.

(TIF)

Figure S4 Founder cell markers appear unaffected in akirin mutant embryos. Wild-type (wt) or akirin mutant embryos (allelic combinations as indicated) were immunostained using antibodies against Even-skipped (stage 11 embryos, panels A-C), Krüppel (late stage 12, panels D-F) and Slouch (late stage 12, panels G-I). Scale bar $=50 \mu \mathrm{m}$.

(TIF)

Figure S5 Comparison of eveMHE-lacZ expression levels in wild-type and akirin mutant embryos. (A) Western blot of whole cell extracts prepared from transgenic wild-type and akirin ${ }^{2}$ mutant embryos carrying the Dmef2-lacZ reporter. Anti- $\alpha$-tubulin staining provided as loading control. Densitometric analysis indicates that $\beta$-galactosidase expression levels are slightly reduced when normalized against tubulin controls ( 0.6 in wild-type versus 0.4 in akirin $^{2}$ mutants). (B,C) Wild type (B) and akirin ${ }^{2}$ mutant $(\mathrm{C})$ embryos carrying a lac $Z$ transgene under the control of the evenskipped MHE element were stained with antibodies against $\beta$ galactosidase. Close-up of two hemisegments presented for comparison. No obvious difference in $\beta$-galactosidase expression was detected in akirin mutants. Scale bar in $(B, C)=20 \mu \mathrm{m}$.

(TIF)

Figure S6 Whole-genomic distribution of Akirin and active transcription markers in polytene chromosomes. Shown are the whole chromosome spreads that are referenced in Figure 4. Scale bar $=5 \mu \mathrm{m}$.

(TIF)

Figure S7 Ectopic overexpression of Twist in 3rd instar salivary glands induces expression of Dmef2. Twist was expressed in salivary glands using the Sgs3-GAL4 driver line. Expression of Dmef2 verified by Western blotting, anti-a-tubulin provided as loading control.

(TIF)

Figure S8 Colocalization of endogenous Akirin protein and expressed Akirin-HA. UAS-Akirin-HA was expressed in larval salivary glands using the Sgs3-GAL4 driver. Polytene chromosomes were prepared and immunostained with antibodies against endogenous Akirin (green) and HA (red). Representative regions of polytene squashes presented. Near-complete colocalization of endogenous Akirin and expressed Akirin-HA was observed (examples shown with white arrows).

(TIF) 
Figure S9 Whole-genomic distribution of Akirin and (A) Brahma, (B) Snrl, and (C) Osa in polytene chromosomes. Shown are the whole chromosome spreads referenced in Figure 5. Scale bar $=5 \mu \mathrm{m}$.

(TIF)

Figure S10 Heterozygous embryos for BRM complex subunit members do not show muscle phenotypes. Stage 16 heterozygote embryos for indicated BRM complex subunit used in Figure 6 were stained with anti-myosin antibodies to show the body wall musculature. Heterozygous embryos were verified by immunostaining for marked balancers; balancer staining channel omitted for clarity. No body wall muscle phenotypes were observed in BRM complex subunit heterozygotes.

(TIF)

Figure S11 Twist and Brahma colocalize in the genome and physically interact. (A) Twist was expressed in salivary glands using the Sgs3-GAL4 driver line. Polytene chromosomes were prepared from Twist-expressing salivary glands and immunostained with antibodies against Twist (red) and Brahma (green). Polytene squashes were costained with DAPI to visualize the DNA. Colocalization between Twist and Brahma (select examples shown in white arrows) was observed in approx. $58 \%$ of gene loci. Scale bar $=5 \mu \mathrm{m}$. Background staining in green channel indicated with (*). (B) Antibodies against the Brahma core subunit successfully immunoprecipitated Twist from wild-type (yw) embryonic extracts. "Input" lanes correspond to $2.5 \%$ of total embryonic extract. Note that (B) is a composite figure; lanes were omitted from the same gel for sake of clarity and direct comparison.

(TIF)

\section{References}

1. Barnes RM, Firulli AB (2009) A twist of insight - the role of Twist-family bHLH factors in development. The International Journal of Developmental Biology 53: 909-924.

2. Yang J, Mani SA, Donaher JL, Ramaswamy S, Itzykson RA, et al. (2004) Twist, a master regulator of morphogenesis, plays an essential role in tumor metastasis. Cell 117: 927-939.

3. Miraoui H, Marie PJ (2010) Pivotal role of Twist in skeletal biology and pathology. Gene 468: 1-7.

4. Baylies MK, Bate M (1996) twist: a myogenic switch in Drosophila. Science 272: 1481-1484.

5. Bernard F, Krejci A, Housden B, Adryan B, Bray SJ (2010) Specificity of Notch pathway activation: Twist controls the transcriptional output in adult muscle progenitors. Development.

6. Castanon I, Baylies MK (2002) A Twist in fate: evolutionary comparison of Twist structure and function. Gene 287: 11-22.

7. Leptin M (1991) twist and snail as positive and negative regulators during Drosophila mesoderm development. Genes \& Development 5: 1568-1576.

8. Sandmann T, Girardot C, Brehme M, Tongprasit W, Stolc V, et al. (2007) A core transcriptional network for early mesoderm development in Drosophila melanogaster. Genes \& Development 21: 436-449.

9. Zeitlinger J, Zinzen RP, Stark A, Kellis M, Zhang H, et al. (2007) Wholegenome ChIP-chip analysis of Dorsal, Twist, and Snail suggests integration of diverse patterning processes in the Drosophila embryo. Genes \& Development 21: 385-390.

10. Castanon I, von Stetina S, Kass J, Baylies MK (2001) Dimerization partners determine the activity of the Twist bHLH protein during Drosophila mesoderm development. Development 128: 3145-3159.

11. Wong M-C, Castanon I, Baylies MK (2008) Daughterless dictates Twist activity in a context-dependent manner during somatic myogenesis. Developmental Biology 317: 417-429.

12. González-Crespo S, Levine M (1993) Interactions between dorsal and helixloop-helix proteins initiate the differentiation of the embryonic mesoderm and neuroectoderm in Drosophila. Genes \& Development 7: 1703-1713.

13. Jiang J, Kosman D, Ip YT, Levine M (1991) The dorsal morphogen gradient regulates the mesoderm determinant twist in early Drosophila embryos. Genes \& Development 5: 1881-1891.

14. Shirokawa JM, Courey AJ (1997) A direct contact between the dorsal rel homology domain and Twist may mediate transcriptional synergy. Molecular and Cellular Biology 17: 3345-3355.

15. Clapier CR, Cairns BR (2009) The Biology of Chromatin Remodeling Complexes. Annual Review of Biochemistry 78: 273-304.
Figure S12 Specificity of antibodies used in chromatin immunoprecipitation assays. Chromatin immunoprecipitations using antibodies against Twist, Akirin and Moira (dark blue) and preimmune antisera (light blue, see Figure 7) were examined for enrichment of the oskar enhancer. No enrichment of the oskar enhancer was detected in immunoprecipitations. To enable direct comparsion, vertical axis in (A), (B), and (C) is identical to corresponding charts in Figure 7. For example, at 2-4 hours, Twist enrichment at the Dmef2 versus oskar enhancers was 0.046 versus 0.0011. For Akirin, occupancy was 0.017 at the Dmef2 enhancer versus 0.00092 at oskar. For Moira, occupancy was 0.012 at the Dmef2 enhancer versus 0.00085 at oskar.

(TIF)

\section{Acknowledgments}

The authors wish to thank J. Treisman, B. Schwer, A. Michelson, G. Mardon, V. Corces, and C. P. Verrijzer for generously sharing fly stocks and reagents. We thank $M$. Hudson and C. Hembree for invaluable technical assistance with qPCR experiments. We thank Flybase, the Bloomington Stock Center, and the DSHB for reagents. We also thank D. Soffar for technical support and members of the Baylies lab for discussion and advice on the manuscript.

\section{Author Contributions}

Conceived and designed the experiments: SJN HA KG YN MKB. Performed the experiments: SJN HA KG. Analyzed the data: SJN HA KG YN MKB. Contributed reagents/materials/analysis tools: SJN HA KG MKB. Wrote the paper: SJN MKB.

16. Mohrmann L, Verrijzer CP (2005) Composition and functional specificity of SWI2/SNF2 class chromatin remodeling complexes. Biochimica et Biophysica Acta 1681: 59-73.

17. Brizuela BJ, Elfring L, Ballard J, Tamkun JW, Kennison JA (1994) Genetic analysis of the brahma gene of Drosophila melanogaster and polytene chromosome subdivisions 72AB. Genetics 137: 803-813.

18. Brizuela BJ, Kennison JA (1997) The Drosophila homeotic gene moira regulates expression of engrailed and HOM genes in imaginal tissues. Mechanisms of Development 65: 209-220.

19. Collins RT, Furukawa T, Tanese N, Treisman JE (1999) Osa associates with the Brahma chromatin remodeling complex and promotes the activation of some target genes. The EMBO journal 18: 7029-7040.

20. Crosby MA, Miller C, Alon T, Watson KL, Verrijzer CP, et al. (1999) The trithorax group gene moira encodes a brahma-associated putative chromatinremodeling factor in Drosophila melanogaster. Molecular and Cellular Biology 19: 1159-1170.

21. Elfring LK, Daniel C, Papoulas O, Deuring R, Sarte M, et al. (1998) Genetic analysis of brahma: the Drosophila homolog of the yeast chromatin remodeling factor SWI2/SNF2. Genetics 148: 251-265.

22. Treisman JE, Luk A, Rubin GM, Heberlein U (1997) evelid antagonizes wingless signaling during Drosophila development and has homology to the Bright family of DNA-binding proteins. Genes \& Development 11: 1949-1962.

23. Armstrong JA, Papoulas O, Daubresse G, Sperling AS, Lis JT, et al. (2002) The Drosophila BRM complex facilitates global transcription by RNA polymerase II The EMBO journal 21: 5245-5254.

24. Chalkley GE, Moshkin YM, Langenberg K, Bezstarosti K, Blastyak A, et al. (2008) The Transcriptional Coactivator SAYP Is a Trithorax Group Signature Subunit of the PBAP Chromatin Remodeling Complex. Molecular and Cellular Biology 28: 2920-2929.

25. Carrera I, Zavadil J, Treisman JE (2008) Two subunits specific to the PBAP chromatin remodeling complex have distinct and redundant functions during drosophila development. Molecular and Cellular Biology 28: 5238-5250.

26. Collins RT, Treisman JE (2000) Osa-containing Brahma chromatin remodeling complexes are required for the repression of wingless target genes. Genes \& Development 14: 3140-3152.

27. Rendina R, Strangi A, Avallone B, Giordano E (2010) Bap170, a Subunit of the Drosophila PBAP Chromatin Remodeling Complex, Negatively Regulates the Egfr Signaling. Genetics.

28. Carrera I, Treisman JE (2008) Message in a nucleus: signaling to the transcriptional machinery. Current Opinion in Genetics \& Development 18: $397-403$. 
29. Goto A, Matsushita K, Gesellchen V, el Chamy L, Kuttenkeuler D, et al. (2008) Akirins are highly conserved nuclear proteins required for NF-kappaBdependent gene expression in drosophila and mice. Nature Immunology 9: 97-104.

30. Marshall A, Salerno MS, Thomas M, Davies T, Berry C, et al. (2008) Mighty is a novel promyogenic factor in skeletal myogenesis. Experimental Cell Research 314: 1013-1029

31. Yu Y, Yussa M, Song J, Hirsch J, Pick L (1999) A double interaction screen identifies positive and negative $\mathrm{ftz}$ gene regulators and ftz-interacting proteins. Mechanisms of Development 83: 95-105.

32. Gonzalez K, Baylies M (2005) bhringi: A novel Twist co-regulator. Program and Abstracts 46th Annual Drosophila Research Conference, San Diego, CA, 2005: 320B

33. Cripps RM, Black BL, Zhao B, Lien CL, Schulz RA, et al. (1998) The myogenic regulatory gene Mef2 is a direct target for transcriptional activation by Twist during Drosophila myogenesis. Genes \& Development 12: 422-434.

34. Yin Z, Xu XL, Frasch M (1997) Regulation of the twist target gene tinman by modular cis-regulatory elements during early mesoderm development. Development 124: 4971-4982.

35. Macqueen DJ, Johnston IA (2009) Evolution of the multifaceted eukaryotic akirin gene family. BMC Evolutionary Biology 9: 34.

36. Bate M (1990) The embryonic development of larval muscles in Drosophila. Development 110: 791-804

37. Chou TB, Perrimon N (1996) The autosomal FLP-DFS technique for generating germline mosaics in Drosophila melanogaster. Genetics 144: 1673-1679.

38. Bulchand S, Menon SD, George SE, Chia W (2010) Muscle wasted: a novel component of the Drosophila histone locus body required for muscle integrity. Journal of Cell Science 123: 2697-2707.

39. Simpson P (1983) Maternal-Zygotic Gene Interactions during Formation of the Dorsoventral Pattern in Drosophila Embryos. Genetics 105: 615-632.

40. Bour BA, O'Brien MA, Lockwood WL, Goldstein ES, Bodmer R, et al. (1995) Drosophila MEF2, a transcription factor that is essential for myogenesis. Genes \& Development 9: 730-741.

41. Gunthorpe D, Beatty KE, Taylor MV (1999) Different levels, but not different isoforms, of the Drosophila transcription factor DMEF2 affect distinct aspects of muscle differentiation. Developmental Biology 215: 130-145.

42. Ranganayakulu G, Zhao B, Dokidis A, Molkentin JD, Olson EN, et al. (1995) A series of mutations in the D-MEF2 transcription factor reveal multiple functions in larval and adult myogenesis in Drosophila. Developmental Biology 171: $169-181$.

43. Elgar SJ, Han J, Taylor MV (2008) mef2 activity levels differentially affect gene expression during Drosophila muscle development. Proceedings of the National Academy of Sciences of the United States of America 105: 918-923.

44. Junion G, Jagla T, Duplant S, Tapin R, Da Ponte J-P, et al. (2005) Mapping Dmef2-binding regulatory modules by using a ChIP-enriched in silico targets approach. Proceedings of the National Academy of Sciences of the United States of America 102: 18479-18484.

45. Lilly B, Galewsky S, Firulli AB, Schulz RA, Olson EN (1994) D-MEF2: a MADS box transcription factor expressed in differentiating mesoderm and muscle cell lineages during Drosophila embryogenesis. Proceedings of the National Academy of Sciences of the United States of America 91: 5662-5666.

46. Nguyen HT, Xu X (1998) Drosophila mef2 expression during mesoderm development is controlled by a complex array of cis-acting regulatory modules. Developmental Biology 204: 550-566.

47. Sandmann T, Jensen LJ, Jakobsen JS, Karzynski MM, Eichenlaub MP, et al. (2006) A temporal map of transcription factor activity: mef2 directly regulates target genes at all stages of muscle development. Developmental Cell 10: 797-807.

48. Taylor MV, Beatty KE, Hunter HK, Baylies MK (1995) Drosophila MEF2 is regulated by twist and is expressed in both the primordia and differentiated cells of the embryonic somatic, visceral and heart musculature. Mechanisms of Development 50: 29-41.

49. Salerno MS, Dyer K, Bracegirdle J, Platt L, Thomas M, et al. (2009) Akirin1 (Mighty), a novel promyogenic factor regulates muscle regeneration and cell chemotaxis. Experimental Cell Research 315: 2012-2021.

50. Spradling A, Penman S, Pardue ML (1975) Analysis of drosophila mRNA by in situ hybridization: sequences transcribed in normal and heat shocked cultured cells. Cell 4: 395-404.

51. Buszczak M, Spradling AC (2006) The Drosophila P68 RNA helicase regulates transcriptional deactivation by promoting RNA release from chromatin. Genes \& Development 20: 977-989.
52. Karam CS, Kellner WA, Takenaka N, Clemmons AW, Corces VG (2010) 14-33 mediates histone cross-talk during transcription elongation in Drosophila. PLoS Genet 6: e1000975. doi:10.1371/journal.pgen.1000975.

53. Nowak SJ, Corces VG (2000) Phosphorylation of histone H3 correlates with transcriptionally active loci. Genes \& Development 14: 3003-3013.

54. Chapman RD, Heidemann M, Albert TK, Mailhammer R, Flatley A, et al (2007) Transcribing RNA polymerase II is phosphorylated at CTD residue serine-7. Science 318: 1780-1782.

55. Thisse C, Thisse B (1992) Dorsoventral development of the Drosophila embryo is controlled by a cascade of transcriptional regulators. Dev Suppl. pp 173-181.

56. Zhimulev IF, Belyaeva ES, Semeshin VF, Shloma VV, Makunin IV, et al. (2003) Overexpression of the SuUR gene induces reversible modifications at pericentric, telomeric and intercalary heterochromatin of Drosophila melanogaster polytene chromosomes. Journal of Cell Science 116: 169-176.

57. Giot L, Bader JS, Brouwer C, Chaudhuri A, Kuang B, et al. (2003) A protein interaction map of Drosophila melanogaster. Science 302: 1727-1736.

58. Möller A, Avila FW, Erickson JW, Jäckle H (2005) Drosophila BAP60 is an essential component of the Brahma complex, required for gene activation and repression. Journal of Molecular Biology 352: 329-337.

59. Halfon MS, Carmena A, Gisselbrecht S, Sackerson CM, Jiménez F, et al. (2000) Ras pathway specificity is determined by the integration of multiple signalactivated and tissue-restricted transcription factors. Cell 103: 63-74.

60. Kim-Ha J, Webster PJ, Smith JL, Macdonald PM (1993) Multiple RNA regulatory elements mediate distinct steps in localization of oskar mRNA. Development 119: 169-178.

61. Celniker SE, Dillon LAL, Gerstein MB, Gunsalus KC, Henikoff S, et al. (2009) Unlocking the secrets of the genome. Nature 459: 927-930.

62. Peña-Rangel MT, Rodriguez I, Riesgo-Escovar JR (2002) A misexpression study examining dorsal thorax formation in Drosophila melanogaster. Genetics 160: 1035-1050.

63. Escudero LM, Caminero E, Schulze KL, Bellen HJ, Modolell J (2005) Charlatan, a $\mathrm{Zn}$-finger transcription factor, establishes a novel level of regulation of the proneural achaete/scute genes of Drosophila. Development 132: $1211-1222$

64. Vorobyeva NE, Soshnikova NV, Nikolenko JV, Kuzmina JL, Nabirochkina EN, et al. (2009) Transcription coactivator SAYP combines chromatin remodeler Brahma and transcription initiation factor TFIID into a single supercomplex. Proceedings of the National Academy of Sciences of the United States of America 106: 11049-11054.

65. Ohkawa Y, Yoshimura S, Higashi C, Marfella CGA, Dacwag CS, et al. (2007) Myogenin and the SWI/SNF ATPase Brgl maintain myogenic gene expression at different stages of skeletal myogenesis. The Journal of Biological Chemistry 282: 6564-6570.

66. Martin SG, Dobi KC, St Johnston D (2001) A rapid method to map mutations in Drosophila. Genome Biology 2: RESEARCH0036.

67. Halfon MS, Gisselbrecht S, Lu J, Estrada B, Keshishian H, et al. (2002) New fluorescent protein reporters for use with the Drosophila Gal4 expression system and for vital detection of balancer chromosomes. Genesis (New York, NY : 2000) 34: 135-138.

68. Brand AH, Perrimon N (1993) Targeted gene expression as a means of altering cell fates and generating dominant phenotypes. Development 118: 401-415.

69. Cox VT, Baylies MK (2005) Specification of individual Slouch muscle progenitors in Drosophila requires sequential Wingless signaling. Development 132: 713-724

70. Richardson BE, Beckett K, Nowak SJ, Baylies MK (2007) SCAR/WAVE and Arp2/3 are crucial for cytoskeletal remodeling at the site of myoblast fusion. Development 134: 4357-4367.

71. Beckett K, Baylies MK (2006) Parcas, a regulator of non-receptor tyrosine kinase signaling, acts during anterior-posterior patterning and somatic muscle development in Drosophila melanogaster. Developmental Biology 299: $176-192$.

72. Nowak SJ, Nahirney PC, Hadjantonakis A-K, Baylies MK (2009) Nap1mediated actin remodeling is essential for mammalian myoblast fusion. Journal of Cell Science 122: 3282-3293.

73. Nowak SJ, Pai G-Y, Corces VG (2003) Protein phosphatase 2A activity affects histone $\mathrm{H} 3$ phosphorylation and transcription in Drosophila melanogaster. Molecular and Cellular Biology 23: 6129-6138.

74. Pfaffl MW (2001) A new mathematical model for relative quantification in realtime RT-PCR. Nucleic Acids Res 29: e45.

75. Lilja T, Aihara H, Stabell M, Nibu Y, Mannervik M (2007) The acetyltransferase activity of Drosophila CBP is dispensable for regulation of the Dpp pathway in the early embryo. Developmental Biology 305: 650-658. 